\title{
Dynamic Key-Aggregate Cryptosystem on Elliptic Curves for Online Data Sharing
}

\author{
Sikhar Patranabis, Yash Shrivastava and Debdeep Mukhopadhyay \\ Department of Computer Science and Engineering \\ Indian Institute of Technology Kharagpur \\ \{sikhar.patranabis, yash.shrivastava, debdeep\}@cse.iitkgp.ernet.in
}

\begin{abstract}
The recent advent of cloud computing and the IoT has made it imperative to have efficient and secure cryptographic schemes for online data sharing. Data owners would ideally want to store their data/files online in an encrypted manner, and delegate decryption rights for some of these to users with appropriate credentials. An efficient and recently proposed solution in this regard is to use the concept of aggregation that allows users to decrypt multiple classes of data using a single key of constant size. In this paper, we propose a secure and dynamic key aggregate encryption scheme for online data sharing that operates on elliptic curve subgroups while allowing dynamic revocation of user access rights. We augment this basic construction to a generalized two-level hierarchical structure that achieves optimal space and time complexities, and also efficiently accommodates extension of data classes. Finally, we propose an extension to the generalized scheme that allows use of efficiently computable bilinear pairings for encryption and decryption operations. Each scheme is formally proven to be semantically secure. Practical experiments have been conducted to validate all claims made in the paper.

Keywords: Key-Aggregate Cryptoystem, Online data sharing, Semantic security, Dynamic access rights
\end{abstract}

\section{Introduction}

The advent of cloud computing and the Internet of Things (IoT) has led to a massive rise in the demand for online data storage and data sharing services. Two very important paradigms that any data sharing service provider must ensure are privacy and flexibility. Since online data almost always resides in shared environments (for instance, multiple virtual machines running on the same physical device), ensuring privacy is a non trivial task. Current technology for secure data sharing comes in two major flavors - trusting a third party auditor [1] or using the user's own key to encrypt her data [2]. Figure 1 describes a realistic online data sharing set-up. Suppose a data owner stores multiple classes of encrypted data online with the intention of providing users decryption keys to one or more such ciphertext classes, based on their respective credentials. She might also wish to dynamically update the delegated access rights based on changes to the data/credibility issues. The challenge therefore is to provide her 


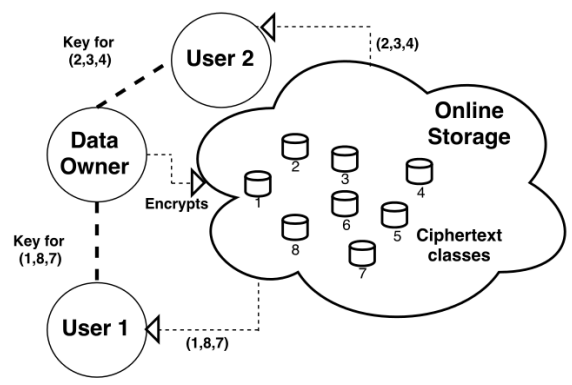

Fig. 1: Example of Online Data Sharing

with a secure and efficient online data sharing scheme that allows updates to user access rights on the fly.

A näive (and extremely inefficient) solution is to have a different decryption key for each ciphertext class, and share them accordingly with users via secured channels. A more efficient proposition is the key-aggregate encryption (KAC) scheme proposed in [3] that combines the power of individual decryption keys, for ciphertext classes in a given subset, into a single key for that subset. This key is specific to the designated subset, meaning that it cannot be used to decrypt any ciphertext class outside that subset. KAC derives its roots from the seminal work by Boneh et.al. [4] that allows broadcasting of data (encrypted by the same public key) among multiple users, each of whom possess their own private keys for decryption. Both these schemes make use of bilinear mappings on multiplicative cyclic groups.

Contributions: In this paper, we propose a basic key-aggregate scheme on additive elliptic subgroups that delegate decryption rights to multiple ciphertext classes using a single constant sized key. The scheme is dynamic in nature, that is, it allows the data owner to revoke access rights of users without having to change the entire set-up, unlike in the existing KAC scheme. We then generalize this scheme into a two-level construction that allows flexible public key extension and maintains constant ciphertext size, while avoiding many of the pitfalls of earlier hierarchical schemes. We provide a formal proof of semantic security for the generalized scheme. We further extend the generalized scheme to allow using popular and efficiently implementable elliptic curve pairing schemes. We compare the time and space requirements of the proposed generalized scheme under various operating configurations. We also compare the performance of our proposed scheme, in terms of key size and resource utilization, with that of other existing schemes in literature.

Organization: The rest of the paper is organized as follows. Section 2 provides a brief overview of state of the art data sharing schemes. Section 3 introduces the notion of key aggregate cryptosystem, and provides a description of the complexity assumptions used to prove the semantic security of our proposed schemes. Our basic dynamic key-aggregate scheme is presented in Section 4. We follow up with a more generalized two-tiered construction of the scheme for efficient public 
key extension in Section 5, and prove its semantic security. A further extension for the generalized scheme that allows using efficiently implementable pairings is introduced and proved semantically secure in Section 6. Experimental results using Tate pairings based implementations of the extended scheme are presented in Section 7. Finally Section 8 concludes the paper.

\section{Related Work}

In this section we present a brief overview of public and private key cryptographic schemes in literature for secure online data sharing. While many of them focus on key aggregation in some form or the other, very few have the ability to provide constant size keys to decrypt an arbitrary number of encrypted entities.

\subsection{Hierarchical Encryption}

One of the most popular techniques for access control in online data storage is to use a pre-defined hierarchy of secret keys [5-8] in the form of a tree-like structure, where access to the key corresponding to any node implicitly grants access to all the keys in the subtree rooted at that node. For instance, [9] uses repeated evaluations of a pseudo-random function/block cipher on a fixed secret to generate a tree hierarchy of symmetric keys. Some more advanced schemes [10-12] extend access control to cyclic and acyclic graphs. A major disadvantage of hierarchical encryption schemes is that granting access to only a selected set of branches within a given subtree warrants an increase in the number of granted secret keys. This in turn blows up the size of the key shared. Thus while hierarchical cryptosystems provide a neat key delegation mechanism when all files in a given branch is to be shared, its efficiency drops drastically as the complexity of the delegation increases.

\subsection{Compact Key Symmetric Encryption}

Compact key encryption for the symmetric key setting has been used in $[13,14]$ to solve the problem of concisely transmitting large number of keys in the broadcast scenario. The basic methodology is to divide the entire ciphertext space into a finite set of classes, followed by a constant size aggregate key generation for the set of classes to be delegated. This scheme thus solves the problem of multi-class delegation faced by hierarchical schemes. However, symmetric key sharing via a secured channel is costly and not always practically viable for many applications on the cloud. Some other schemes in the symmetric key setting also attempt to reduce the key size [15], but they are not aimed at decryption key delegation and are hence not very relevant to the present discussion.

\subsection{Compact Key Identity-Based Encryption}

Identity-Based Encryption (IBE) is a public key-based encryption scheme in which the public key for any user is an identity-string corresponding to that 
user. Proposed initially in [16], IBE was concretized by the proposition of two very widely cited and popular IBEs - The Boneh-Franklin scheme [17] and Cocks' encryption scheme [18]. An IBE system comprises of a trusted private key generator that holds a master-secret key and issues a secret key to each user based on the user identity. Each user receives a message that has been encrypted using her id and some public parameters, and can decrypt the same using the secret key allotted to her by the trusted party. Compact key IBEs have been proposed in [19] and [20]. The former approach involves the use of random oracles while the latter shuns the use of oracles. Both these schemes allow aggregation of keys; however each key must come from a different identity division. Fuzzy IBE [21] allows for a single compact key to decrypt multiple ciphertexts, but they must have been encrypted under a closed set of identities, and the scheme does not work in practical scenarios for arbitrary identities.

\subsection{Attribute Based Encryption}

Attribute-based encryption (ABE) [22-24] allows each user to be identified by a set of attributes. An encrypted file stored in cloud can only be decrypted by an user who has access to the corresponding secret key. The secret key is securely transmitted to the user who satisfies the access control policies set by the data owner. A major drawback of this scheme is that each time the access right to a particular user is revoked the entire ciphertext has to be recrypted in the cloud. The idea of ABE has been extended to shared keys for user groups in [25] with the focus on collusion resistance and not on key size compression.

\subsection{Proxy Re-Encryption}

Proxy re-encryption is another technique to achieve fine-grained access control and scalable user revocation in unreliable clouds [26]. In this method the data owner and a semi trusted proxy cloud share a secret key in advance, with which the cloud can be delegated to re-encrypt data on behalf of the data owner. The semi-trusted proxy re-encrypts the data using the data owner's public key, thus converting it into a file that can in turn be decrypted by the secret key of the client. In the whole process, the proxy has no knowledge of the data being sent. An extension to this technique has been proposed in [27] that allows the cloud servers to automatically re-encrypt data based on their internal clocks, without any external trigger. However, proxy re-encryption essentially transfers the responsibility for secure key storage from the delegatee to the proxy and is susceptible to collusion attacks. It is also important to ensure that the transformation key of the proxy is well protected, and every decryption would require a separate interaction with the proxy, which is inconvenient for applications on the cloud.

\subsection{Key-Aggregate Cryptosystems (KAC)}

The authors of [3] proposes an efficient scheme, namely KAC, that allows secure and efficient sharing of data on the cloud. The scheme is a public-key cryp- 
tosystem that uses constant size ciphertexts such that efficient delegation of decryption rights for any set of ciphertexts are possible. When a user demands for a particular subset of the available classes of data, the data owner computes an aggregate key which integrates the power of the individual decryption keys corresponding to each class of data. However, KAC as proposed in [3] suffers from three major drawbacks, each of which we address in this paper. First of all, the security assumption of KAC seems to be the Bilinear Diffie Hellman Exponent (BDHE) assumption [28]; however no concrete proofs of semantic security are provided by the authors in [3]. Secondly, with respect to user access rights, $\mathrm{KAC}$ is a static scheme in the sense that once a user is in possession of the aggregate key corresponding to a subset of files from data owner, the owner cannot dynamically revoke the permission of the client for accessing one or more updated files. Since dynamic changes in access rights is extremely common in online data storage, this scenario needs to be tackled. Finally, the public key extension of KAC proposed in [3] is extremely cumbersome and resource consuming since registration of each new public key-private key pair requires the number of classes to be extended by the original number of classes.

\section{Preliminaries}

We begin by formally defining the Key Aggregate Cryptosystem (KAC), and stating the complexity assumptions used to prove the security of the encryption schemes proposed in this paper.

\subsection{The Key Aggregate Cryptosystem (KAC)}

A key aggregate cryptosystem is an ensemble of the following randomized algorithms:

1. $\operatorname{Setup}\left(1^{\lambda}, n\right)$ : Takes as input the number of ciphertext classes $n$ and the group order parameter $\lambda$. Outputs the public parameter $P K$. Also computes a secret parameter $t$ used for encryption which is not made public. It is only known to data owners with credentials to control client access rights.

2. Keygen(): Outputs the public and master-secret key pair :

$(P K=\gamma P, m s k=\gamma)$.

3. $\operatorname{Encrypt}(P K, i, m)$ : Takes as input the public key parameter $P K$, the ciphertext class $i$ and the message $m$. Outputs the ciphertext $\mathcal{C}$ corresponding to the message $m$ belonging to class $i$.

4. $\operatorname{Extract}(m s k=\gamma, \mathcal{S})$ : Takes as input the master secret key $\gamma$ and a subset $\mathcal{S} \subset\{1,2, \cdots, n\}$. Computes the aggregate key $K_{\mathcal{S}}$ and the dynamic access control parameter $U$. The tuple $\left(K_{\mathcal{S}}, U\right)$ is transmitted via a secure channel to users that have access rights to $\mathcal{S}$.

5. Decrypt $\left(K_{\mathcal{S}}, U, \mathcal{S}, i, \mathcal{C}=\left\{c_{1}, c_{2}, c_{3}\right\}\right)$ : Takes as input the aggregate key $K_{\mathcal{S}}$ corresponding to a subset $\mathcal{S} \subset\{1,2, \cdots, n\}$, the dynamic access parameter $U$, the ciphertext class $i$ and the ciphertext $\mathcal{C}$. Outputs the decrypted message $m$. 


\subsection{Semantic Security of KAC}

We now define the semantic security of a key-aggregate encryption system against an adversary using the following game between an attack algorithm $\mathcal{A}$ and a challenger $\mathcal{B}$. Both $\mathcal{A}$ and $\mathcal{B}$ are given $n$, the total number of ciphertext classes, as input. The game proceeds through the following stages.

1. Init: Algorithm $\mathcal{A}$ begins by outputting a set $\mathcal{S} \subset\{1,2, \cdots, n\}$ of receivers that it wishes to attack. For each ciphertext class $i \in \mathcal{S}$, challenger $\mathcal{B}$ performs the SetUp-i, Challenge-i and Guess-i steps. Note that the number of iterations is polynomial in $|S|$.

2. SetUp-i: Challenger $\mathcal{B}$ generates the public param, public key $P K$, the access parameter $U$, and provides them to $\mathcal{A}$. In addition, $\mathcal{B}$ also generates and furnishes $\mathcal{A}$ with the aggregate key $K_{\overline{\mathcal{S}}}$ that allows $\mathcal{A}$ to decrypt any ciphertext class $j \notin \mathcal{S}$.

3. Challenge-i: Challenger $\mathcal{B}$ performs an encryption of the secret message $m_{i}$ belonging to the $i^{\text {th }}$ class to obtain the ciphertext $\mathcal{C}$. Next, $\mathcal{B}$ picks a random $b \in(0,1)$. It sets $K_{b}=m_{i}$ and picks a random $K_{1-b}$ from the set of possible plaintext messages. It then gives $\left(\mathcal{C}, K_{0}, K_{1}\right)$ to algorithm $\mathcal{A}$ as a challenge.

4. Guess-i: The adversary $\mathcal{A}$ outputs a guess $b^{\prime}$ of $b$. If $b^{\prime}=b, \mathcal{A}$ wins and the challenger $\mathcal{B}$ loses. Otherwise, the game moves on to the next ciphertext class in $\mathcal{S}$ until all ciphertext classes in $\mathcal{S}$ are exhausted.

If the adversary $\mathcal{A}$ fails to predict correctly for all ciphertext classes in $\mathcal{S}$, only then $\mathcal{A}$ loses the game. Let $A d v K A C_{\mathcal{A}, n}$ denote the probability that $\mathcal{A}$ wins the game when the challenger is given $n$ as input. We say that a key-aggregate encryption system is $(\tau, \epsilon, n)$ semantically secure if for all $\tau$-time algorithms $\mathcal{A}$ we have that $\left|A d v K A C_{\mathcal{A}, n}-\frac{1}{2}\right|<\epsilon$ where $\epsilon$ is a very small quantity. Note that the adversary $\mathcal{A}$ is non-adaptive; it chooses $\mathcal{S}$, and obtains the aggregate decryption key for all ciphertext classes outside of $\mathcal{S}$, before it even sees the public parameters param or the public key $P K$.

\subsection{The Complexity Assumptions}

We now introduce the complexity assumptions used in this paper. In this section, we make several references to bilinear non-degenerate mappings on elliptic curve sub-groups, popularly known in literature as pairings. Hence it seems logical to provide a brief background on bilinear pairing based schemes on elliptic curve sugroups.

Bilinear Pairings: We present a brief outline of the necessary facts about bilinear pairings on elliptic curves that are used in the forthcoming discussion. Let $\mathbb{K}=F_{p}$ be a field of prime order $p$, and let an elliptic curve over $\mathbb{K}$ be defined by the Weierstrass [28] equation:

$$
E(\mathbb{K}): y^{2}+a_{1} x y+a_{3} y=x^{3}+a_{2} x^{2}+a_{4} x+a_{6}
$$


where $a_{1}, a_{2}, a_{3}, a_{4}, a_{5} \in \mathbb{K}$. The curve must be non-singular. In particular, if $\operatorname{char}(\mathbb{K}) \neq 2,3$, the equation takes the special form $y^{2}=x^{3}+a_{4} x+a_{6}$ with $4 a_{4}{ }^{3}+27 a_{6}{ }^{2} \neq 0$. Let $\bar{K}=F_{p^{k}}$ be the smallest extension field of $K=F_{p}$ that contains the $q^{\text {th }}$ roots of unity. Here, $k$ is called the embedding degree with respect to $K$ and $q$. We denote the set of $q$-torsion points on the elliptic curve as $E(\bar{K})[q]$ ( $q$-torsion points essentially have order $q$ ).

A pairing is a bilinear map defined over elliptic curve subgroups. Let $\mathbb{G}_{1}$ and $\mathbb{G}_{2}$ be two such additive cyclic subgroups of the same prime order $q$ and let $\mathbb{G}_{T}$ be a multiplicative group, also of order $q$ with identity element 1 . Let $P$ and $Q$ be generators for $\mathbb{G}_{1}$ and $\mathbb{G}_{2}$ respectively. A pairing $\hat{e}^{\prime}: \mathbb{G}_{1} \times \mathbb{G}_{2} \longrightarrow \mathbb{G}_{T}$ satisfying the following the following properties is said to be a bilinear mapping.

- Bilinear: $\forall P_{1}, P_{2} \in \mathbb{G}_{1}, Q_{1}, Q_{2} \in \mathbb{G}_{2}$, and $a, b \in \mathbb{Z}$, we have the following:

$$
\begin{aligned}
& \hat{e}^{\prime}\left(a P_{1}, b Q_{1}\right)=\hat{e}^{\prime}\left(P_{1}, Q_{1}\right)^{a b} \\
& \hat{e^{\prime}}\left(P_{1}+P_{2}, Q_{1}\right)=\hat{e^{\prime}}\left(P_{1}, Q_{1}\right) \hat{e^{\prime}}\left(P_{2}, Q_{1}\right) \\
& \hat{e}^{\prime}\left(P_{1}, Q_{1}+Q_{2}\right)=\hat{e^{\prime}}\left(P_{1}, Q_{1}\right) \hat{e^{\prime}}\left(P_{1}, Q_{2}\right)
\end{aligned}
$$

- Non-degeneracy: If for all $P_{i} \in \mathbb{G}_{1}, \hat{e^{\prime}}\left(P_{1}, Q_{1}\right)=1$ then $Q_{1}=$. Alternatively, if $P$ and $Q$ be the generators for $\mathbb{G}_{1}$ and $\mathbb{G}_{2}$ respectively where neither group only contains the point at infinity, then $\hat{e}^{\prime}(P, Q) \neq 1$

- Computability: There exists an efficient algorithm to compute $\hat{e}^{\prime}(R, S) \forall R \in$ $\mathbb{G}_{1}, S \in \mathbb{G}_{2}$

It is important to note that $\mathbb{G}_{1}$ and $\mathbb{G}_{2}$ could be identical groups as well.

The First Complexity Assumption: Our first complexity assumption is the $l$-BDHE problem [4] in a bilinear elliptic curve subgroup $\mathbb{G}$, defined as follows. Given a vector of $2 l+1$ elements $\left(H, P, \alpha P, \alpha^{2} P, \cdots, \alpha^{l} P, \alpha^{l+2} P \cdots, \alpha^{2 l} P\right) \in$ $\mathbb{G}^{2 l+1}$ as input, and a bilinear pairing $\hat{e}^{\prime}: \mathbb{G}_{1} \times \mathbb{G}_{1} \longrightarrow \mathbb{G}_{T}$ output $\hat{e}^{\prime}(P, H)^{\alpha^{l+1}} \in$ $\mathbb{G}_{T}$. Since $\alpha^{l+1} P$ is not an input, the bilinear pairing is of no real use in this regard. Using the shorthand $P_{i}=\alpha^{i} P$, an algorithm $\mathcal{A}$ is said to have an advantage $\epsilon$ in solving $l$-BDHE if

$\operatorname{Pr}\left[\mathcal{A}\left(H, P, P_{1}, P_{2}, \cdots, P_{l}, P_{l+2} \cdots, P_{2 l}\right)=\hat{e^{\prime}}\left(P_{l+1}, H\right)\right] \geq \epsilon$, where the probability is over the random choice of $H, P \in \mathbb{G}$, random choice of $\alpha \in \mathbb{Z}_{q}$ and random bits used by $\mathcal{A}$. The decisional version of $l$-BDHE for elliptic curve subgroups may be analogously defined. Let $Y_{(P, \alpha, l)}=\left(P, P_{1}, P_{2}, \cdots, P_{l}, P_{l+2} \cdots, P_{2 l}\right)$. An algorithm $\mathcal{B}$ that outputs $b \in\{0,1\}$ has advantage $\epsilon$ in solving decisional $l$-BDHE in $\mathbb{G}$ if $\left|\operatorname{Pr}\left[\mathcal{B}\left(P, H, Y_{(P, \alpha, l)}, \hat{e}^{\prime}\left(P_{l+1}, H\right)\right)=0\right]-\operatorname{Pr}\left[\mathcal{B}\left(G, H, Y_{(P, \alpha, l)}, T\right)=0\right]\right| \geq \epsilon$, where the probability is over the random choice of $H, P \in \mathbb{G}$, random choice of $\alpha \in \mathbb{Z}_{q}$, random choice of $T \in \mathbb{G}_{T}$ and random bits used by $\mathcal{B}$. We refer to the left and right probability distributions as $L$-BDHE and $R$-BDHE respectively. Thus, it can be said that the decision $(\tau, \epsilon, l)$-BDHE assumption for elliptic curves holds in $\mathbb{G}$ if no $\tau$-time algorithm has advantage $\epsilon$ in solving the decisional $l$-BDHE problem over elliptic curve subgroup $\mathbb{G}$. 
The Second Complexity Assumption: We next define the $(l, l)$-BDHE problem over a pair of equi-prime order bilinear elliptic curve subgroups $\mathbb{G}_{1}$ with generator $P$ and $\mathbb{G}_{2}$ with generator $Q$. Given a vector of $3 l+2$ elements $\left(H, P, Q, \alpha P, \alpha^{2} P, \cdots, \alpha^{l} P, \alpha^{l+2} P \cdots, \alpha^{2 l} P, \alpha Q, \alpha^{2} Q, \cdots, \alpha^{l} Q\right)$ as input, where $P$ and $\alpha^{i} P \in \mathbb{G}_{1}$ and $H, Q, \alpha^{i} Q \in \mathbb{G}_{2}$, along with a bilinear pairing $e^{\prime \prime}$ : $\mathbb{G}_{1} \times \mathbb{G}_{2} \longrightarrow \mathbb{G}_{T}$, output $\hat{e^{\prime}}(P, H)^{\alpha^{l+1}} \in \mathbb{G}_{T}$. Since $\alpha^{l+1} P$ is not an input, the bilinear pairing is of no real use in this regard. Using the shorthand $P_{i}=\alpha^{i} P$ and $Q_{i}=\alpha^{i} Q$, an algorithm $\mathcal{A}$ is said to have an advantage $\epsilon$ in solving $(l, l)$-BDHE if $\operatorname{Pr}\left[\mathcal{A}\left(H, P, Q, P_{1}, P_{2}, \cdots, P_{l}, P_{l+2} \cdots, P_{2 l}, Q_{1}, \cdots, Q_{l}\right)=\hat{e}^{\prime}\left(P_{l+1}, H\right)\right] \geq \epsilon$ where the probability is over the random choice of $P \in \mathbb{G}_{1}, H, Q \in \mathbb{G}_{2}$, random choice of $\alpha \in \mathbb{Z}_{q}$ and random bits used by $\mathcal{A}$. We may also define the decisional $(l, l)$-BDHE problem over elliptic curve subgroup pairs as follows. Let $Y_{(P, \alpha, l)}=$ $\left(P, P_{1}, P_{2}, \cdots, P_{l}, P_{l+2} \cdots, P_{2 l}\right)$ and $Y_{(Q, \alpha, l)}^{\prime}=\left(Q, Q_{1}, Q_{2}, \cdots, Q_{l}\right)$. Also let $H$ be a random element in $\mathbb{G}_{2}$. An algorithm $\mathcal{B}$ that outputs $b \in\{0,1\}$ has advantage $\epsilon$ in solving decisional $l$-BDHE in $\mathbb{G}$ if $\mid \operatorname{Pr}\left[\mathcal{B}\left(P, Q, H, Y_{(P, \alpha, l)}, Y_{(Q, \alpha, l)}^{\prime}, \hat{e}^{\prime}\left(P_{l+1}, H\right)\right)\right.$ $=0]-\operatorname{Pr}\left[\mathcal{B}\left(G, H, Y_{(P, \alpha, l)}, Y_{(Q, \alpha, l)}^{\prime}, T\right)=0\right] \mid \geq \epsilon$, where the probability is over the random choice of $P \in \mathbb{G}_{1}, H, Q \in \mathbb{G}_{2}$, random choice of $\alpha \in \mathbb{Z}_{q}$, random choice of $T \in \mathbb{G}_{T}$ and random bits used by $\mathcal{B}$. We refer to the left and right probability distributions as $L^{\prime}$-BDHE and $R^{\prime}$-BDHE respectively. Thus, it can be said that the decision $(\tau, \epsilon, l, l)$-BDHE assumption for elliptic curves holds in $\left(\mathbb{G}_{1}, \mathbb{G}_{2}\right)$ if no $\tau$-time algorithm has advantage $\epsilon$ in solving the decisional $(l, l)$-BDHE problem over elliptic curve subgroups $\mathbb{G}_{1}$ and $\mathbb{G}_{2}$. To the best of our knowledge, the $(l, l)$-BDHE problem has not been introduced in literature before.

Proving the Validity of the Second Complexity Assumption: We prove here that the decision $(\tau, \epsilon, l, l)$-BDHE assumption for elliptic curves holds in equi-prime order subgroups $\left(\mathbb{G}_{1}, \mathbb{G}_{2}\right)$ if the decision $(\tau, \epsilon, l)$-BDHE assumption for elliptic curves holds in $\mathbb{G}_{1}$. Let $\hat{e}^{\prime}: \mathbb{G}_{1} \times \mathbb{G}_{1} \longrightarrow \mathbb{G}_{T}$ and $\hat{e}^{\prime \prime}: \mathbb{G}_{1} \times \mathbb{G}_{2} \longrightarrow \mathbb{G}_{T}$ be bilinear pairings. Also, let $P$ and $Q$ are the generators for $\mathbb{G}_{1}$ and $\mathbb{G}_{2}$ respectively. We first make the following observation.

Observation 1: Since $G_{1}$ and $G_{2}$ have the same prime order (say $q$ ), there exists a bijection $\varphi: \mathbb{G}_{1} \longrightarrow \mathbb{G}_{2}$ such that $\varphi(a P)=a Q$ for all $a \in \mathbb{Z}_{q}$. Similarly, since $\mathbb{G}_{T}$ also has order $q$, there also exists a mapping $\phi: \mathbb{G}_{T} \longrightarrow \mathbb{G}_{T}$ such that $\phi\left(\hat{e^{\prime}}\left(H_{1}, H_{2}\right)\right)=\hat{e^{\prime \prime}}\left(H_{1}, \varphi\left(H_{2}\right)\right)$ for all $H_{1}, H_{2} \in \mathbb{G}_{1}$.

Let $\mathcal{A}$ be a $\tau$-time adversary that has advantage greater than $\epsilon$ in solving the decision $(l, l)$-BDHE problem over equi-prime order subgroups $\left(\mathbb{G}_{1}, \mathbb{G}_{2}\right)$. We build an algorithm $\mathcal{B}$ that has advantage at least $\epsilon$ in solving the $l$-BDHE problem in $\mathbb{G}_{1}$. Algorithm $\mathcal{B}$ takes as input a random $l$-BDHE challenge $\left(P, H, Y_{(P, \alpha, l)}, Z\right)$ where $Z$ is either $\hat{e}^{\prime}\left(P_{l+1}, H\right)$ or a random value in $\mathbb{G}_{T} \cdot \mathcal{B}$ computes $Y_{Q, \alpha, l}^{\prime}$ by setting $Q_{i}=\varphi\left(P_{i}\right)$ for $i=1,2, \cdots, l$. $\mathcal{B}$ also computes $H^{\prime}=\varphi(H) \in$ $\mathbb{G}_{2}$ and $Z^{\prime}=\phi(Z) \in \mathbb{Z}$. then randomly chooses a bit $b \in(0,1)$ and sets $T_{b}$ as $Z^{\prime}$ and $T_{1-b}$ as a random element in $\mathbb{G}_{T}$. The challenge given to $\mathcal{A}$ is $\left(\left(P, Q, H^{\prime}, Y_{(P, \alpha, l)}, Y_{Q, \alpha, l}^{\prime}\right), T_{0}, T_{1}\right)$. Quite evidently, when $Z=\hat{e}^{\prime}\left(P_{l+1}, H\right)$ (i.e. the input to $\mathcal{B}$ is a $l$-BDHE tuple), then $\left(\left(P, Q, H^{\prime}, Y_{(P, \alpha, l)}, Y_{Q, \alpha, l}^{\prime}\right), T_{0}, T_{1}\right)$ is a valid challenge to $A$. This is because in such a case, $T_{b}=Z^{\prime}=\phi(Z)=$ 
$\phi\left(\hat{e}^{\prime}\left(P_{l+1}, H\right)\right)=\hat{e}^{\prime \prime}\left(P_{l+1}, H^{\prime}\right)$. On the other hand, if $Z$ is a random element in $\mathbb{G}_{T}$ (i.e. the input to $\mathcal{B}$ is a random tuple), then $T_{0}$ and $T_{1}$ are just random independent elements of $\mathbb{G}_{T}$.

Now, $\mathcal{A}$ outputs a guess $b^{\prime}$ of $b$. If $b^{\prime}=b, \mathcal{B}$ outputs 0 (indicating that $\left.Z=\hat{e}^{\prime}\left(P_{l+1}, H\right)\right)$. Otherwise, it outputs 1 (indicating that $Z$ is random in $\mathbb{G}_{T}$ ). A simple analysis reveals that if $\left(P, H, Y_{(P, \alpha, l)}, Z\right)$ is sampled from $R$-BDHE, $\operatorname{Pr}\left[\mathcal{B}\left(G, H, Y_{(P, \alpha, l)}, Z\right)=0\right]=\frac{1}{2}$, while if $\left(P, H, Y_{(P, \alpha, l)}, Z\right)$ is sampled from $L$ BDHE, $\left|\operatorname{Pr}\left[\mathcal{B}\left(G, H, Y_{(P, \alpha, l)}, Z\right)\right]-\frac{1}{2}\right| \geq \epsilon$. So, the probability that $\mathcal{B}$ outputs correctly is at least $\epsilon$, which in turn implies that $\mathcal{B}$ has advantage at least $\epsilon$ in solving the $l$-BDHE problem. This concludes the proof.

\section{The Proposed Dynamic Key-Aggregate Cryptosystem: The Basic Case}

In this section, we present the design of our proposed dynamic key-aggregate storage scheme on additive elliptic curve subgroups assuming that there are $n$ ciphertext classes. Our scheme ensures that the ciphertext and aggregate key are of constant size, while the public parameter size is linear in the number of ciphertext classes. Unlike the scheme proposed in [3], the proposed scheme allows dynamic revocation of user access rights without having to massively change the system parameters. We also present a proof of security for the proposed scheme.

\subsection{The Basic Construction of Dynamic KAC}

Let $\mathbb{G}$ be an additive cyclic elliptic curve subgroup of prime order $q$, where $2^{\lambda} \leq q \leq 2^{\lambda+1}$, such that the point $P$ is a generator for $\mathbb{G}$. Also, let $\mathbb{G}_{T}$ be a multiplicative group of order $q$ with identity element 1 . We assume that there exists an efficiently computable bilinear pairing $\hat{e}^{\prime}: \mathbb{G} \times \mathbb{G} \longrightarrow \mathbb{G}_{T}$. We now present the basic construction of our proposed key-aggregate encryption scheme.

The scheme consists of the following five phases.

1. $\operatorname{Setup}\left(1^{\lambda}, n\right)$ : Randomly pick $\alpha \in \mathbb{Z}_{q}$. Compute $P_{i}=\alpha^{i} P \in \mathbb{G}$ for $i=$ $1, \cdots, n, n+2, \cdots, 2 n$. Output the system parameter as

param $=\left(P, P_{1}, \cdots, P_{n}, P_{n+2}, \cdots, P_{2 n}\right)$. The system also randomly chooses a secret parameter $t \in \mathbb{Z}_{q}$ which is not made public. It is only known to data owners with credentials to control client access rights.

2. Keygen(): Pick $\gamma \in \mathbb{Z}_{q}$, output the public and master-secret key pair : $(P K=\gamma P, m s k=\gamma)$.

3. $\operatorname{Encrypt}(P K, i, m)$ : For a message $m \in \mathbb{G}_{T}$ and an index $i \in\{1,2, \cdots, n\}$, randomly choose $r \in \mathbb{Z}_{q}$ and let $t^{\prime}=t+r \in \mathbb{Z}_{q}$. Then the ciphertext is computed as

$\mathcal{C}=\left(r P, t^{\prime}\left(P K+P_{i}\right), m \cdot \hat{e^{\prime}}\left(P_{n}, t^{\prime} P_{1}\right)\right)=\left(c_{1}, c_{2}, c_{3}\right)$

4. $\operatorname{Extract}(m s k=\gamma, \mathcal{S})$ : For the set $\mathcal{S}$ of indices $j$ the aggregate key is computed as

$K_{\mathcal{S}}=\sum_{j \in \mathcal{S}} \gamma P_{n+1-j}=\sum_{j \in \mathcal{S}} \alpha^{n+1-j} P K$ 
and the dynamic access control parameter $U$ is computed as $t P$. Thus the net aggregate key is $\left(K_{\mathcal{S}}, U\right)$ which is transmitted via a secure channel to users that have access rights to $\mathbb{S}$.

5. Decrypt $\left(K_{\mathcal{S}}, U, \mathcal{S}, i, \mathcal{C}=\left\{c_{1}, c_{2}, c_{3}\right\}\right)$ : If $i \notin \mathcal{S}$, output $\perp$. Otherwise return the message

$$
\hat{m}=c_{3} \hat{e^{\prime}}\left(K_{\mathcal{S}}+\sum_{j \in \mathcal{S}, j \neq i} P_{n+1-j+i}, U+c_{1}\right) /\left(\hat{e^{\prime}}\left(\sum_{j \in \mathcal{S}} P_{n+1-j}, c_{2}\right)\right) .
$$

The proof of correctness of this scheme is presented below.

$$
\begin{aligned}
& \hat{m}=c_{3} \frac{\hat{e^{\prime}}\left(K_{\mathcal{S}}+\sum_{j \in \mathcal{S}, j \neq i} P_{n+1-j+i}, U+c_{1}\right)}{\hat{e}^{\prime}\left(\sum_{j \in \mathcal{S}} P_{n+1-j}, c_{2}\right)} \\
& =c_{3} \frac{\hat{e}^{\prime}\left(\sum_{j \in \mathcal{S}} \gamma P_{n+1-j}+\sum_{j \in \mathcal{S}, j \neq i} P_{n+1-j+i}, t^{\prime} P\right)}{\hat{e}^{\prime}\left(\sum_{j \in \mathcal{S}} P_{n+1-j}, t^{\prime}\left(P K+P_{i}\right)\right)} \\
& =c_{3} \frac{\hat{e^{\prime}}\left(\sum_{j \in \mathcal{S}} \gamma P_{n+1-j}, t^{\prime} P\right) \hat{e^{\prime}}\left(\sum_{j \in \mathcal{S}}\left(P_{n+1-j+i}\right)-P_{n+1}, t^{\prime} P\right)}{\left.\hat{e}^{\prime}\left(\sum_{j \in \mathcal{S}} P_{n+1-j}, t^{\prime} P K\right) \hat{e}^{\prime}\left(\sum_{j \in \mathcal{S}} P_{n+1-j}, t^{\prime} P_{i}\right)\right)} \\
& =c_{3} \frac{\hat{e^{\prime}}\left(\sum_{j \in \mathcal{S}} P_{n+1-j+i}, t^{\prime} P\right)}{\left.\hat{e}^{\prime}\left(P_{n+1}, t^{\prime} P\right) \hat{e}^{\prime}\left(\sum_{j \in \mathcal{S}} P_{n+1-j}, t^{\prime} P_{i}\right)\right)} \\
& =c_{3} \frac{\hat{e^{\prime}}\left(\sum_{j \in \mathcal{S}} P_{n+1-j+i}, t^{\prime} P\right)}{\left.\hat{e}^{\prime}\left(P_{n+1}, t^{\prime} P\right) \hat{e}^{\prime}\left(\sum_{j \in \mathcal{S}} P_{n+1-j+i}, t^{\prime} P\right)\right)} \\
& =m \frac{\hat{e}^{\prime}\left(P_{n}, t^{\prime} P_{1}\right)}{\hat{e}^{\prime}\left(P_{n+1}, t^{\prime} P\right)} \\
& =m
\end{aligned}
$$

\subsection{Dynamic Access Control}

An important aspect of the proposed scheme is the fact that it allows the data owner to dynamically update user access permissions. In KAC [3], once the data owner issues an aggregate key corresponding to a set of ciphertext classes to a user, revoking the user's access permissions to the same is not possible without changing the master secret key. However, changing the master secret key each time an user's access privileges to a ciphertext class need to be updated, is a very expensive option and may not be practically feasible. Our scheme, on the other hand, offers a solution to this problem by allowing the data owner to dynamically update user access privileges.

We achieve this by making the parameter $U=t P$ a part of the aggregate key in our proposed scheme and not a part of the ciphertext. The user must have the correct value of $U$ in possession to be able to decrypt any encrypted ciphertext class in the subset $\mathcal{S}$. Now suppose the data owner wishes to alter the access rights to the subset $\mathcal{S}$. She can simply re-encrypt all ciphertexts in that class using a different random element $\hat{t} \in \mathbb{Z}_{q}$, and then provide the updated dynamic access parameter $\hat{U}=\hat{t} P$ to only those users who she wishes to delegate access to. The decrypted value will give the correct message $m$ only if the same $t$ is used for both encryption and decryption. This is a major difference between our scheme and the scheme proposed in [3], where the knowledge of the random 
parameter was only embedded as part of the ciphertext itself, and could not be used to control access rights of users. Moreover, since $U$ is of constant size and needs to be transmitted only when changed (and not for every encryption), there is no significant degradation in performance.

\subsection{Performance and Efficiency}

The decryption time for any subset of ciphertext classes $\mathcal{S}$ is essentially dominated by the computation of $W_{\mathcal{S}}=\sum_{j \in \mathcal{S}} P_{n+1-j+i}$. However, if a user has already computed $\sum_{j \in \mathcal{S}^{\prime}} P_{n+1-j+i}$ for a subset $S^{\prime}$ similar to $S$, then she can easily compute the desired value by at most $\left|\mathcal{S}-\mathcal{S}^{\prime}\right|$ operations. For similar subsets $S$ and $S^{\prime}$, this value is expected to be fairly small. A suggested in [4], for subsets of very large size $(n-r, r \ll n)$, an advantageous approach could be to pre-compute $\sum_{j=1}^{j=n} P_{n+1-j+i}$ corresponding to $i=1$ to $n$, which would allow the user to decrypt using only $r$ group operations, and would require only $r$ elements of param. Similar optimizations would also hold for the encryption operation where pre-computation of $\sum_{j=1}^{j=n} P_{n+1-j}$ is useful for large subsets.

It is important to note that our proposed scheme fixes the number of ciphertext classes beforehand, thus limiting the scope for ciphertext class extension. The only way to increase the number of classes is to change the public key parameters, which would therefore require some kind of administrative privileges, and cannot be done by an user for her own purposes. However, in online data sharing environments, users may wish to register their own public key-private key pairs for new ciphertext classes according to their own requirements. Such an extension to the scheme would make extremely convenient and attractive to potential users. A proposal made in [3] recommends that the user be allowed to register new public-private key pairs, at the cost of increasing the number of ciphertext classes by $n$ each time. This is both impractical and wasteful. In the next section, we present a two-tier generalization of our scheme that tackles this issue in a more economical fashion. We provide a proof of semantic security for the base case presented here. As will be shown later, this proof is a special case of the proof for the generalized scheme presented in the next section.

\subsection{Formal Proof of Semantic Security}

In this section we formally prove the security of the proposed generalized keyaggregate encryption scheme.

The Reduced Scheme: The ciphertext $\mathcal{C}=\left(c_{1}, c_{2}, c_{3}\right)$ output by the Encypt operation essentially embeds the value of $m$ in $c_{3}$ by multiplying it with $\hat{e}^{\prime}\left(P_{n}, t^{\prime} P_{1}\right)$. The main secret is thus $\hat{e}^{\prime}\left(P_{n}, t^{\prime} P_{1}\right)=\hat{e}^{\prime}\left(P_{n+1}, t^{\prime} P\right)$, the knowledge of which is transmitted using $\left(c_{1}, c_{2}\right)$, and is decrypted using the aggregate key $K_{\mathcal{S}}$ and the dynamic access parameter $U$. Consequently, the security of our proposed scheme is equivalent to that of a reduced key-aggregate encryption scheme that 
simply uses the reduced ciphertext $\left(c_{1}, c_{2}\right)$, the aggregate key $K_{\mathcal{S}}$ and the dynamic access parameter $U$ to successfully transmit and decrypt the value of $\hat{e}^{\prime}\left(P_{n+1}, t^{\prime} P\right)$. We prove the semantic security of this reduced scheme parameterized with a given number of ciphertext classes $n$, which also amounts to proving the semantic security of our original encryption scheme for the same number of ciphertext classes.

The Adversarial Model: We make the following assumptions about the adversary $\mathcal{A}$ :

1. The adversary has the aggregate key that allows her to access any ciphertext class other than those in the target subset $\mathcal{S}$, that is, she possesses $K_{\overline{\mathcal{S}}}$.

2. The adversary has access to the public parameters param and $P K$, and also possesses the dynamic access parameter $U$.

The Proof of Security: The security proof presented here uses the first complexity assumption stated in 3.3. Let $\mathbb{G}$ be a bilinear elliptic curve subgroup of prime order $q$ and $G_{T}$ be a multiplicative group of order $q$. Let $\hat{e}^{\prime}: \mathbb{G} \times \mathbb{G} \longrightarrow \mathbb{G}_{T}$ be a bilinear non-degenerate pairing. We claim that for any pair of positive integers $n, n^{\prime}\left(n^{\prime}>n\right)$, the reduced key-aggregate encryption scheme over elliptic curve subgroups is $\left(\tau, \epsilon, n^{\prime}\right)$ semantically secure if the decision $(\tau, \epsilon, n)$-BDHE assumption holds in $\mathbb{G}$.

Proof: Let for a given input $n^{\prime}, \mathcal{A}$ be a $\tau$-time adversary that has advantage greater than $\epsilon$ in solving the reduced scheme parameterized with a fixed $n$. Using $\mathcal{A}$, we build an algorithm $\mathcal{B}$ that has advantage at least $\epsilon$ in solving the $n$ BDHE problem in $\mathbb{G}$. Algorithm $\mathcal{B}$ takes as input a random $n$-BDHE challenge $\left(P, H, Y_{(P, \alpha, n)}, Z\right)$ (where $Z$ is either $\hat{e}^{\prime}\left(P_{n+1}, H\right)$ or a random value in $\mathbb{G}_{T}$ ), and proceeds as follows.

1. Init: $\mathcal{B}$ runs $\mathcal{A}$ and receives the set $\mathcal{S}$ of ciphertext classes that $\mathcal{A}$ wishes to be challenged on. For each ciphertext class $i \in \mathcal{S}, \mathcal{B}$ performs the SetUpi, Challenge-i and Guess-i steps. Note that the number of iterations is polynomial in $|S|$.

2. SetUp-i: $\mathcal{B}$ should generate the public param, public key $P K$, the access parameter $U$, and the aggregate key $K_{\overline{\mathcal{S}}}$ and provide them to $\mathcal{A}$. They are generated as follows.

- param is set as $\left(P, Y_{P, \alpha, n}\right)$.

- $P K$ is set as $u P-P_{i}$ where $u$ is randomly chosen from $\mathbb{Z}_{q}$.

$-K_{\overline{\mathcal{S}}}$ is set as $\sum_{j \notin \mathcal{S}}\left(u P_{n+1-j}-\left(P_{n+1-j+i}\right)\right)$. Note that $K_{\overline{\mathcal{S}}}$ is equal to $\sum_{j \notin \mathcal{S}} \alpha^{n+1-j} P K$, in accordance with the specification provided by the scheme. Moreover, $\mathcal{B}$ is aware that $i \notin \overline{\mathcal{S}}$ (implying $i \neq j$ ), and hence has all the resources to compute $K_{\overline{\mathcal{S}}}$.

- $U$ is set as some random element in $\mathbb{G}$.

Since $P, \alpha, U$ and the $u$ values are chosen uniformly at random, the public parameters and the public key have an identical distribution to that in the actual construction. 
3. Challenge-i: To generate the challenge for the ciphertext class $i, \mathcal{B}$ computes $\left(c_{1}, c_{2}\right)$ as $(H-U, u H)$. It then randomly chooses a bit $b \in(0,1)$ and sets $T_{b}$ as $Z$ and $T_{1-b}$ as a random element in $\mathbb{G}_{T}$. The challenge given to $\mathcal{A}$ is $\left(\left(c_{1}, c_{2}\right), T_{0}, T_{1}\right)$. We claim that when $Z=\hat{e}^{\prime}\left(P_{n+1}, H\right)$ (i.e. the input to $\mathcal{B}$ is a $n$-BDHE tuple), then $\left(\left(c_{1}, c_{2}\right), T_{0}, T_{1}\right)$ is a valid challenge to $A$. Since $P$ is a generator of $\mathbb{G}, H=t^{\prime} P$ for some $t^{\prime} \in \mathbb{Z}_{q}$, resulting in the following.

- $U=t P$ for some $t \in \mathbb{Z}_{q}$

- $c_{1}=H-U=\left(t^{\prime}-t\right) P=r P$ where $r=t^{\prime}-t$

$-c_{2}=u H=u t^{\prime} P=t^{\prime}(u P)=t^{\prime}\left(u P-P_{i}+P_{i}\right)=t^{\prime}\left(P K+P_{i}\right)$

- $K_{b}=Z=\hat{e}^{\prime}\left(P_{n+1}, H\right)=\hat{e}^{\prime}\left(P_{n+1}, t^{\prime} P\right)$

On the other hand, if $Z$ is a random element in $\mathbb{G}_{T}$ (i.e. the input to $\mathcal{B}$ is a random tuple), then $K_{0}$ and $K_{1}$ are just random independent elements of $\mathbb{G}_{T}$.

4. Guess-i: The adversary $\mathcal{A}$ outputs a guess $b^{\prime}$ of $b$. If $b^{\prime}=b, \mathcal{B}$ outputs 0 (indicating that $Z=\hat{e}^{\prime}\left(P_{n+1}, H\right)$ ), and terminates. Otherwise, it goes for the next ciphertext class in $\mathcal{S}$.

If $\mathcal{A}$ returns $b^{\prime} \neq b$ for each ciphertext class $i \in \mathcal{S}$. In the latter case, $\mathcal{B}$ outputs 1 (indicating that $Z$ is random in $\mathbb{G}_{T}$ ). We now analyze the probability that $\mathcal{B}$ gives a correct output. If $\left(P, H, Y_{(P, \alpha, n)}, Z\right)$ is sampled from $R$-BDHE, $\operatorname{Pr}\left[\mathcal{B}\left(G, H, Y_{(P, \alpha, n)}, Z\right)=0\right]=\frac{1}{2}$, while if $\left(P, H, Y_{(P, \alpha, n)}, Z\right)$ is sampled from $L$-BDHE, $\left|\operatorname{Pr}\left[\mathcal{B}\left(G, H, Y_{(P, \alpha, n)}, Z\right)\right]-\frac{1}{2}\right| \geq \epsilon$. So, the probability that $\mathcal{B}$ outputs correctly is at least $1-(1-\epsilon)^{|\mathcal{S}|} \geq \epsilon$, implying that $\mathcal{B}$ has advantage at least $\epsilon$ in solving the $n$-BDHE problem in $\mathbb{G}$. This concludes the proof.

\section{A Generalized Version of Dynamic KAC}

In this section, we focus on building an efficiently extensible version of our proposed scheme that allows an user to economically increase the number of ciphertext classes while registering a new public key-private key pair. We adopt the idea presented in [4] to develop a hierarchical structure that has multiple instances (say $n_{1}$ ) of the original scheme running in parallel. Each such instance in turn provides locally aggregate keys for $n_{2}$ ciphertext sub-classes. Each ciphertext class thus now has a double index $\left(i_{1}, i_{2}\right)$ where $1 \leq i_{1} \leq n_{1}$ and $1 \leq i_{2} \leq n_{2}$. This allows the overall setup to handle $n=n_{1} n_{2}$ classes. However, it is important to note that all the instances can use the same public parameters. This interaction among the instances helps to largely improve performance. We further point out that while in [4], the generalized construction offers a tradeoff between the public parameter size and the ciphertext size, our generalized scheme actually reduces the public parameter size without compromising on the size of the ciphertext. Further, addition of a single new key increases the number of classes only by $n_{2}$ and not by $n$. Setting $n_{2} \ll n$ thus achieves significant improvement in performance over the existing proposal. 


\subsection{The Construction of the Generalized KAC}

Let $n_{2}$ be a fixed positive integer. Our proposed $n_{2}$-generalized key-aggregate encryption scheme over elliptic curve subgroups is as described below. It may be noted that the bilinear additive elliptic curve sub-group $\mathbb{G}$ and the multiplicative group $\mathbb{G}_{T}$, as well as the pairing $\hat{e}^{\prime}$ are the same as in the basic scheme. The algorithm sets up $n_{1}=\left\lfloor n / n_{2}\right\rfloor$ instances of the basic scheme, each of which handles $n_{2}$ ciphertext classes. The original scheme is thus a special case of the extended scheme with $n_{1}=1$ and $n_{2}=n$.

1. $\operatorname{Setup}\left(1^{\lambda}, n_{2}\right)$ : Randomly pick $\alpha \in \mathbb{Z}_{q}$. Compute $P_{i}=\alpha^{i} P \in \mathbb{G}$ for $i=$ $1, \cdots, n_{2}, n_{2}+2, \cdots, 2 n_{2}$. Output the system parameter as

param $=\left(P, P_{1}, \cdots, P_{n_{2}}, P_{n_{2}+2}, \cdots, P_{2 n_{2}}\right)$. The system randomly chooses a secret parameter $t \in \mathbb{Z}_{q}$ which is not made public. It is only known to data owners with credentials to control client access rights.

2. Keygen(): Pick $\gamma_{1}, \gamma_{2}, \cdots, \gamma_{n_{1}} \in \mathbb{Z}_{q}$, output the public and master-secret key pair :

$\left(P K=\left(p k_{1}, p k_{2}, \cdots, p k_{n_{1}}\right)=\left(\gamma_{1} P, \gamma_{2} P, \cdots, \gamma_{n_{1}} P\right), m s k=\left(\gamma_{1}, \gamma_{2}, \cdots, \gamma_{n_{1}}\right)\right)$.

3. $\operatorname{Encrypt}\left(p k_{i_{1}},\left(i_{1}, i_{2}\right), m\right)$ : For a message $m \in \mathbb{G}_{T}$ and an index $\left(i_{1}, i_{2}\right) \in$ $\left\{1,2, \cdots, n_{1}\right\} \times\left\{1,2, \cdots, n_{2}\right\}$, randomly choose $r \in \mathbb{Z}_{q}$ and let $t^{\prime}=t+r \in$ $\mathbb{Z}_{q}$. Then compute the ciphertext $\mathcal{C}=\left(r P, t^{\prime}\left(p k_{i_{1}}+P_{i_{2}}\right), m \cdot \hat{e^{\prime}}\left(P_{n_{2}}, t^{\prime} P_{1}\right)\right)=$ $\left(c_{1}, c_{2}, c_{3}\right)$.

4. $\operatorname{Extract}(m s k=\gamma, \mathcal{S})$ : For the set $\mathcal{S}$ of indices $\left(j_{1}, j_{2}\right)$ the aggregate key is computed as $K_{\mathcal{S}}=\left(k_{\mathcal{S}}^{1}, k_{\mathcal{S}}^{2}, \cdots, k_{\mathcal{S}}^{n_{1}}\right)=$ $\left(\sum_{\left(1, j_{2}\right) \in \mathcal{S}} \gamma_{1} P_{n_{2}+1-j_{2}}, \sum_{\left(2, j_{2}\right) \in \mathcal{S}} \gamma_{2} P_{n_{2}+1-j_{2}}, \cdots, \sum_{\left(n_{1}, j_{2}\right) \in \mathcal{S}} \gamma_{n_{1}} P_{n_{2}+1-j_{2}}\right)$ and the dynamic access control parameter $U$ is computed as $t P$. Thus the net aggregate key is $\left(K_{\mathcal{S}}, U\right)$ which is transmitted via a secure channel to users that have access rights to $\mathbb{S}$. Note that $k_{\mathcal{S}}^{j_{1}}=\sum_{\left(j_{1}, j_{2}\right) \in \mathcal{S}} \alpha^{n+1-j} p k_{j_{1}}$ for $j_{1}=1,2, \cdots, n_{1}$.

5. $\operatorname{Decrypt}\left(K_{\mathcal{S}}, U, \mathcal{S},\left(i_{1}, i_{2}\right), \mathcal{C}=\left\{c_{1}, c_{2}, c_{3}\right\}\right)$ : If $\left(i_{1}, i_{2}\right) \notin \mathcal{S}$, output $\perp$. Otherwise return the message

$$
\hat{m}=c_{3} \frac{\hat{e}^{\prime}\left(k_{\mathcal{S}}^{i_{1}}+\sum_{\left(i_{1}, j_{2}\right) \in \mathcal{S}, j_{2} \neq i_{2}} P_{n_{2}+1-j_{2}+i_{2}}, U+c_{1}\right)}{\hat{e}^{\prime}\left(\sum_{\left(i_{1}, j_{2}\right) \in \mathcal{S}} P_{n_{2}+1-j_{2}}, c_{2}\right)} .
$$

The proof of correctness for the generalized scheme is presented below. 


$$
\begin{aligned}
\hat{m} & =c_{3} \frac{\hat{e}^{\prime}\left(k_{\mathcal{S}}^{i_{1}}+\sum_{\left(i_{1}, j_{2}\right) \in \mathcal{S}, j_{2} \neq i_{2}} P_{n_{2}+1-j_{2}+i_{2}}, U+c_{1}\right)}{\hat{e}^{\prime}\left(\sum_{\left(i_{1}, j_{2}\right) \in \mathcal{S}} P_{n_{2}+1-j_{2}}, c_{2}\right)} \\
& =c_{3} \frac{\hat{e}^{\prime}\left(\sum_{\left(i_{1}, j_{2}\right) \in \mathcal{S}} \gamma_{i_{1}} P_{n_{2}+1-j_{2}}+\sum_{\left(i_{1}, j_{2}\right) \in \mathcal{S}, j_{2} \neq i_{2}} P_{n_{2}+1-j_{2}+i_{2}}, t^{\prime} P\right)}{\hat{e}^{\prime}\left(\sum_{\left(i_{1}, j_{2}\right) \in \mathcal{S}} P_{n_{2}+1-j_{2}}, t^{\prime}\left(p k_{i_{1}}+P_{i_{2}}\right)\right.} \\
& =c_{3} \frac{\hat{e}^{\prime}\left(\sum_{\left(i_{1}, j_{2}\right) \in \mathcal{S}} \gamma_{i_{1}} P_{n_{2}+1-j_{2}}, t^{\prime} P\right) \hat{e}^{\prime}\left(\sum_{\left(i_{1}, j_{2}\right) \in \mathcal{S}}\left(P_{n_{2}+1-j_{2}+i_{2}}\right)-P_{n_{2}+1}, t^{\prime} P\right)}{\hat{e}^{\prime}\left(\sum_{\left(i_{1}, j_{2}\right) \in \mathcal{S}} P_{n_{2}+1-j_{2}}, t^{\prime} p k_{i_{1}}\right) \hat{e}^{\prime}\left(\sum_{\left(i_{1}, j_{2}\right) \in \mathcal{S}} P_{n_{2}+1-j_{2}}, t^{\prime} P_{i_{2}}\right)} \\
& =c_{3} \frac{\hat{e^{\prime}}\left(\sum_{\left(i_{1}, j_{2}\right) \in \mathcal{S}} P_{n_{2}+1-j_{2}+i_{2}}, t^{\prime} P\right)}{\hat{e}^{\prime}\left(P_{n_{2}+1}, t^{\prime} P\right) \hat{e}^{\prime}\left(\sum_{\left(i_{1}, j_{2}\right) \in \mathcal{S}} P_{n_{2}+1-j_{2}}, t^{\prime} P_{i_{2}}\right)} \\
& =c_{3} \frac{\hat{e}^{\prime}\left(\sum_{\left(i_{1}, j_{2}\right) \in \mathcal{S}} P_{n_{2}+1-j_{2}+i_{2}}, t^{\prime} P\right)}{\hat{e}^{\prime}\left(P_{n_{2}+1}, t^{\prime} P\right) \hat{e}^{\prime}\left(\sum_{\left(i_{1}, j_{2}\right) \in \mathcal{S}} P_{n_{2}+1-j_{2}+i_{2}}, t^{\prime} P\right)} \\
& =m \frac{\hat{e}^{\prime}\left(P_{n_{2}}, t^{\prime} P_{1}\right)}{\hat{e}^{\prime}\left(P_{n_{2}+1}, t^{\prime} P\right)} \\
& =m
\end{aligned}
$$

\subsection{Semantic Security of the Generalized KAC}

The Reduced Generalized Scheme: As in the original scheme, we may analogously define a reduced version of the generalized encryption scheme. We note that once again, in the generalized scheme, the ciphertext $\mathcal{C}=\left(c_{1}, c_{2}, c_{3}\right)$ output by the Encypt operation essentially embeds the value of $m$ in $c_{3}$ by multiplying it with $\hat{e}^{\prime}\left(P_{n_{2}}, t P_{1}\right)$. Consequently, the security of our proposed scheme is equivalent to that of a reduced generalized key-aggregate encryption scheme that simply uses the reduced ciphertext $\left(c_{1}, c_{2}\right)$, the aggregate key $K_{\mathcal{S}}$ and the dynamic access parameter $U$ to successfully transmit and decrypt the value of $\hat{e^{\prime}}\left(P_{n_{2}}, t^{\prime} P_{1}\right)=\hat{e^{\prime}}\left(P_{n_{2}+1}, t^{\prime} P\right)$. We prove the semantic security of this reduced scheme parameterized with a given number of ciphertext classes $n_{2}$ for each instance, which also amounts to proving the semantic security of our original encryption scheme for the same number of ciphertext classes. Note that the proof of security is independent of the number of instances $n_{1}$ that run in parallel. The adversarial model is the same as in the case of the proof for the basic scheme.

The Security Proof: The security proof presented here uses the first complexity assumption stated in 3.3. Let $\mathbb{G}$ be a bilinear elliptic curve subgroup of prime order $q$ and $G_{T}$ be a multiplicative group of order $q$. Let $\hat{e}^{\prime}: \mathbb{G} \times \mathbb{G} \longrightarrow \mathbb{G}_{T}$ be a bilinear non-degenerate pairing. For any pair of positive integers $n_{2}, n^{\prime}\left(n^{\prime}>n_{2}\right)$ our proposed $n_{2}$-generalized reduced key-aggregate encryption scheme over elliptic curve subgroups is $\left(\tau, \epsilon, n^{\prime}\right)$ semantically secure if the decision $\left(\tau, \epsilon, n_{2}\right)$-BDHE assumption holds in $\mathbb{G}$. We now prove this statement below.

Proof: Let for a given input $n^{\prime}, \mathcal{A}$ be a $\tau$-time adversary that has advantage greater than $\epsilon$ for the reduced scheme parameterized with a given $n_{2}$. We build an algorithm $\mathcal{B}$ that has advantage at least $\epsilon$ in solving the $n_{2}$-BDHE problem in 
$\mathbb{G}$. Algorithm $\mathcal{B}$ takes as input a random $n_{2}$-BDHE challenge $\left(P, H, Y_{\left(P, \alpha, n_{2}\right)}, Z\right)$ where $Z$ is either $\hat{e^{\prime}}\left(P_{n_{2}+1}, H\right)$ or a random value in $\mathbb{G}_{T}$. Algorithm $\mathcal{B}$ proceeds as follows.

1. Init: Algorithm $\mathcal{B}$ runs $\mathcal{A}$ and receives the set $\mathcal{S}$ of ciphertext classes that $\mathcal{A}$ wishes to be challenged on. For each ciphertext class $\left(i_{1}, i_{2}\right) \in \mathcal{S}, \mathcal{B}$ performs the SetUp- $\left(\mathbf{i}_{1}, \mathbf{i}_{2}\right)$, Challenge- $\left(\mathbf{i}_{1}, \mathbf{i}_{2}\right)$ and Guess- $\left(\mathbf{i}_{1}, \mathbf{i}_{2}\right)$ steps. Note that the number of iterations is polynomial in $|S|$.

2. SetUp- $\left(\mathbf{i}_{1}, \mathbf{i}_{\mathbf{2}}\right): \mathcal{B}$ should generate the public param, public key $P K$, the access parameter $U$, and the aggregate key $K_{\overline{\mathcal{S}}}$. For the iteration corresponding to ciphertext class $\left(i_{1}, i_{2}\right)$, they are generated as follows.

- param is set as $\left(P, Y_{P, \alpha, n_{2}}\right)$.

- Randomly generate $u_{1}, u_{2}, \cdots, u_{n_{1}} \in \mathbb{Z}_{q}$. Then, set $P K=$ $\left(p k_{1}, p k_{2}, \cdots, p k_{n_{1}}\right)$, with $p k_{j_{1}}=u_{j_{1}} P-P_{i_{2}}$ for $j_{1}=1,2, \cdots, n_{1}$.

- Set $K_{\overline{\mathcal{S}}}=\left(k_{\mathcal{S}}^{1}, k_{\mathcal{S}}^{2}, \cdots, k_{\mathcal{S}}^{n_{1}}\right)$, where $k_{\mathcal{S}}^{j_{1}}$ is set as $\sum_{\left(j_{1}, j_{2}\right) \notin \mathcal{S}}\left(u P_{n_{2}+1-j_{2}}-\right.$ $\left.\left(P_{n_{2}+1-j_{2}+i_{2}}\right)\right)$. Then, $k_{\mathcal{S}}^{j_{1}}=\sum_{\left(j_{1}, j_{2}\right) \notin \mathcal{S}} \alpha^{n_{2}+1-j_{2}} p k_{j_{1}}$, which is as per the scheme specification. Note that $\mathcal{B}$ knows that $\left(i_{1}, i_{2}\right) \notin \overline{\mathcal{S}}$, and hence has all the resources to compute this aggregate key for $\overline{\mathcal{S}}$.

- $U$ is set as some random element in $\mathbb{G}$.

Note that since $P, \alpha, U$ and the $u_{j_{1}}$ values are chosen uniformly at random, the public key has an identical distribution to that in the actual construction.

3. Challenge- $\left(\mathbf{i}_{1}, \mathbf{i}_{2}\right)$ : To generate the challenge for the ciphertext class $\left(i_{1}, i_{2}\right)$, $\mathcal{B}$ computes $\left(c_{1}, c_{2}\right)$ as $\left(H-U, u_{i_{1}} H\right)$. It then randomly chooses a bit $b \in(0,1)$ and sets $K_{b}$ as $Z$ and $K_{1-b}$ as a random element in $\mathbb{G}_{T}$. The challenge given to $\mathcal{A}$ is $\left(\left(c_{1}, c_{2}\right), K_{0}, K_{1}\right)$.

We claim that when $Z=\hat{e}^{\prime}\left(P_{n_{2}+1}, H\right)$ (i.e. the input to $\mathcal{B}$ is a $n_{2}$-BDHE tuple), then $\left(\left(c_{1}, c_{2}\right), K_{0}, K_{1}\right)$ is a valid challenge to $A$. We prove this claim here. we point out that $P$ is a generator of $\mathbb{G}$ and so $H=t^{\prime} P$ for some $t^{\prime} \in \mathbb{Z}_{q}$. Putting $H$ as $t^{\prime} P$ gives us the following:

- $U=t P$ for some $t \in \mathbb{Z}_{q}$

$-c_{1}=H-U=\left(t^{\prime}-t\right) P=r P$ for $r=t^{\prime}-t$

$-c_{2}=u_{i_{1}} H=\left(u_{i_{1}}\right) t^{\prime} P=t^{\prime}\left(u_{i_{1}} P\right)=t^{\prime}\left(u_{i_{1}} P-P_{i_{2}}+P_{i_{2}}\right)=t^{\prime}\left(p k_{i_{1}}+P_{i_{2}}\right)$

$-K_{b}=Z=\hat{e^{\prime}}\left(P_{n_{2}+1}, H\right)=\hat{e^{\prime}}\left(P_{n_{2}+1}, t^{\prime} P\right)$

On the other hand, if $Z$ is a random element in $\mathbb{G}_{T}$ (i.e. the input to $\mathcal{B}$ is a random tuple), then $K_{0}$ and $K_{1}$ are just random independent elements of $\mathbb{G}_{T}$.

4. Guess- $\left(\mathbf{i}_{1}, \mathbf{i}_{\mathbf{2}}\right)$ : The adversary $\mathcal{A}$ outputs a guess $b^{\prime}$ of $b$. If $b^{\prime}=b, \mathcal{B}$ outputs 0 (indicating that $Z=\hat{e^{\prime}}\left(P_{n_{2}+1}, H\right)$ ), and terminates. Otherwise, it goes for the next ciphertext class in $\mathcal{S}$.

If after $|\mathcal{S}|$ iterations, $b^{\prime} \neq b$ for each ciphertext class $\left(i_{1}, i_{2}\right) \in \mathcal{S}$, the algorithm $\mathcal{B}$ outputs 1 (indicating that $Z$ is random in $\mathbb{G}_{T}$ ). We now analyze the probability that $\mathcal{B}$ gives a correct output. If $\left(P, H, Y_{\left(P, \alpha, n_{2}\right)}, Z\right)$ is sampled from $R$-BDHE, $\operatorname{Pr}\left[\mathcal{B}\left(G, H, Y_{\left(P, \alpha, n_{2}\right)}, Z\right)=0\right]=\frac{1}{2}$, while if $\left(P, H, Y_{\left(P, \alpha, n_{2}\right)}, Z\right)$ is sampled from $L$-BDHE, $\left|\operatorname{Pr}\left[\mathcal{B}\left(G, H, Y_{\left(P, \alpha, n_{2}\right)}, Z\right)\right]-\frac{1}{2}\right| \geq \epsilon$. So, the probability that $\mathcal{B}$ outputs 
Table 1: Comparison between the Basic and Generalized schemes

\begin{tabular}{|c|c|c|c|}
\hline Item & Nature of Computation & Original scheme & Generalized scheme \\
\hline \hline param $($ SetUp $)$ & One-time & $\mathcal{O}(n)$ & $\mathcal{O}\left(n_{2}\right)$ \\
\hline PK $($ KeyGen $)$ & One-time & $\mathcal{O}(1)$ & $\mathcal{O}\left(n_{1}\right)$ \\
\hline$K_{\mathcal{S}}$ Extract) & One-time & $\mathcal{O}(1)$ & $\mathcal{O}\left(n_{1}\right)$ \\
\hline $\mathcal{C}$ & One per Message & $\mathcal{O}(1)$ & $\mathcal{O}(1)$ \\
\hline Encrypt & One Per Message & $\mathcal{O}(1)$ & $\mathcal{O}(|\mathcal{S}|)$ \\
\hline Decrypt & One Per Message & $\mathcal{O}(|\mathcal{S}|)$ & \multicolumn{3}{|l}{} \\
\hline \hline
\end{tabular}

correctly is at least $1-\left(\frac{1}{2}-\epsilon\right)^{|\mathcal{S}|} \geq \frac{1}{2}+\epsilon$. Thus $\mathcal{B}$ has advantage at least $\epsilon$ in solving the $n_{2}$-BDHE problem. This concludes the proof. Note that the instance of this proof with $n_{1}=1$ and $n_{2}=n$ serves as the proof of security for the basic $K A C$ scheme proposed in Section 4.

Performance Trade off with the Basic Scheme: We compare the various parameter sizes for the proposed original and extended schemes in table 1. We note that SetUp and KeyGen are both one-time operations, and for a given subset $\mathcal{S}$, the Extract operation is also performed once to generate the corresponding aggregate key $K_{\mathcal{S}}$. The most important advantage that the generalized scheme provides is the user's ability to efficiently extend the number of ciphertext classes. As far as encryption and decryption are concerned, encryption should ideally take the same time for both schemes, while decryption is actually expected to be faster for the generalized construction as $n_{2} \leq n$.

\subsection{A Flexible Extension Policy}

If a user needs to classify her ciphertexts into more that $n$ classes, she can register for additional key pairs $\left(p k_{n_{1}+1}, m s k_{n_{1}+1}\right), \cdots,\left(p k_{n_{1}+l}, m s k_{n_{1}+l}\right)$ as per her requirements. Each new key registration increases the number of classes by $n_{2}$, where $n_{2} \leq n$. The idea of under-utilization stems from the fact that registration of each public-private key pair increases the number of classes by $n_{2}$. However, it is not necessary that all the existing classes are utilized at any given point of time. For instance, a user may at any point of time want to register $l$ new privatepublic key pairs, however she will in all probability not use up all $l n_{2}$ additional classes of messages that could be encrypted using the newly registered keys. We stress here is that, unlike in the public key extension scheme proposed in [3] where the values of $n_{1}$ and $n_{2}$ are fixed to 1 and $n$ respectively, our generalized construction provides a choice of $n_{1}$ and $n_{2}$ so that the system administrator could choose pair of values suited to their requirements.

We propose a metric to quantify the under-utilization of ciphertext classes for a given configuration of the system. Let us assume that at some instance of time, there are $n_{1}+l$ private-public key pairs registered in the system, and $c_{i}$ classes corresponding to each key are being utilized. We define the utilization coefficient as $\frac{1}{1+\xi}$, where $\xi=-\frac{1}{n_{1}} \sum_{i=1 c_{i} \neq 0}^{n_{1}} \log \left(\frac{c_{i}}{n_{2}}\right)$. An efficient scheme tries to minimize the value of $\xi$ to achieve good utilization of the existing set of classes. 


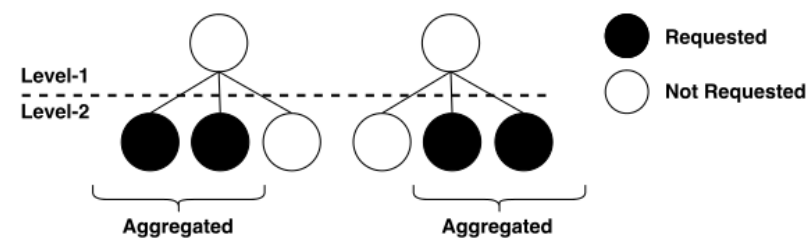

Fig. 2: A Practical Request Scenario in the Hierarchical Setting

The value is maximum when $c_{i}=n_{2} \forall i=1,2, \cdots, n_{2}$. Note that $c_{i}=0$ implies that no subclasses under the given key $p k_{i}$ are being utilized, which is equivalent to not registering the key at all.

To stress the importance of the flexible extension policy, we provide a simplified example here. We consider two possible configurations of the extended scheme. In the first configuration, $n_{1}=1$ and $n_{2}=n$, which is essentially identical to the public key extension scheme proposed in [3]. The other configuration has $n_{1}>1$ and $n_{2}<n$. Now assume that before extension, both schemes utilized $c$ ciphertext classes out of the $n$ possible classes, equally distributed across all key pairs. Now suppose a situation arises where an user needs to register $l$ more key pairs, and utilizes $z<n_{2}$ classes corresponding to each key. In the first configuration, we have $\xi_{1}=-\frac{1}{l+1}\left(l \log \left(\frac{z}{n}\right)+\log \left(\frac{c}{n}\right)\right)$, while for the second configuration, $\xi_{2}=-\frac{1}{l+n_{1}}\left(l\left(\log \left(\frac{z}{n_{2}}\right)\right)+n_{1} \log \left(\frac{c}{n}\right)\right)$. Now for $l>\left(\frac{n_{1}}{\log n_{1}}-1\right) \log \left(\frac{z}{c}\right)-1$, $\xi_{2}<\xi_{1}$. Thus for any value of $\left(n_{1}, n_{2}\right)$ other than $(1, n)$, there exists a value of $l$ for which the scheme achieves better utilization coefficient. Since $l$ is expected to increase in a dynamic scenario, our public key extension scheme eventually performs better than the scheme suggested in [3].

\subsection{Advantage over Hierarchical Encryption Based Schemes}

Although the generalized scheme has a two level hierarchy (with each of the $n_{1}$ parallely executing instances of the basic scheme representing a node in the top level and the actual ciphertext classes representing nodes in the lower level), it avoids the pitfalls of existing hierarchical encryption based schemes $[5,8]$. In standard tree based hierarchical systems, granting access to the key corresponding to any node implicitly grants access to all the keys in the subtree rooted at that node. This means granting access to a selected set of nodes in a given subtree would blow up the key-size to be the same as the number of nodes. This is avoided in our generalized scheme, since any number of nodes (ciphertext classes) that belong to the same instance may be aggregated into a single key. Figure 2 summarizes this phenomenon. In the situation depicted, a tree-based hierarchy system would require 4 decryption keys, while our scheme would require only 2 . In this respect, our scheme has similar advantages to that of [3]. 


\section{Extending the Generalized KAC for Efficient Pairings on Elliptic Curve Subgroups}

The encryption schemes proposed so far use the assumption that the elliptic curve pairing bilinear pairing $\hat{e}^{\prime}: \mathbb{G}_{1} \times \mathbb{G}_{1} \longrightarrow \mathbb{G}_{T}$ satisfies the property $\hat{e}^{\prime}(P, P) \neq 1$, where $P$ is the generator for $G_{1}$. In this section, we propose an extension to the generalized $n_{2}$-scheme that allows using pairings of the form $e^{\prime \prime}: \mathbb{G}_{1} \times \mathbb{G}_{2} \longrightarrow \mathbb{G}_{T}$, where $G_{1}$ and $G_{2}$ are two elliptic curve subgroups of the same prime order. The motivation behind this extension is that many popular pairing algorithms such as the Tate [29], Eta [30], and Ate [31] pairings are defined over two distinct elliptic curve subgroups $G_{1}$ and $G_{2}$ of the same order. Many efficient implementations of such pairings on sensor nodes such as TinyTate [32] have been proposed in literature. This motivates us to modify our scheme in a manner that allows using such well-known pairings. The modified encryption scheme described below allows using a pairing $e^{\prime \prime}: \mathbb{G}_{1} \times \mathbb{G}_{2} \longrightarrow \mathbb{G}_{T}$ with $P$ generator of $G_{1}$ and $Q$ generator of $G_{2}$.

\subsection{Construction of the Extended KAC}

1. $\operatorname{Setup}\left(1^{\lambda}, n_{2}\right)$ : Randomly pick $\alpha \in \mathbb{Z}_{q}$. Compute $P_{i}=\alpha^{i} P \in \mathbb{G}_{1}$ for $i=$ $1, \cdots, n_{2}, n_{2}+2, \cdots, 2 n_{2}$ and $Q_{i}=\alpha^{i} Q \in \mathbb{G}_{2}$ for $i=1, \cdots, n_{2}$. Output the system parameter as

param $=\left(P, P_{1}, \cdots, P_{n_{2}}, P_{n_{2}+2}, \cdots, P_{2 n_{2}}, Q, Q_{1}, \cdots, Q_{n_{2}}\right)$. The system also randomly chooses secret parameters $t \in \mathbb{Z}_{q}$ which is not made public. It is only transferred through a secure channel to data owners with credentials to control client access rights.

2. Keygen(): Pick $\gamma_{1}, \gamma_{2}, \cdots, \gamma_{n_{1}} \in \mathbb{Z}_{q}$, output the public and master-secret key tuple:

$$
\begin{aligned}
& \left(P K^{1}=\left(p k^{1}{ }_{1}, p k^{1}{ }_{2}, \cdots, p k^{1}{ }_{n_{1}}\right)=\left(\gamma_{1} P, \gamma_{2} P, \cdots, \gamma_{n_{1}} P\right), P K^{2}=\right. \\
& \left.\left(p k^{2}{ }_{1}, p k^{2}{ }_{2}, \cdots, p k^{2}{ }_{n_{1}}\right)=\left(\gamma_{1} Q, \gamma_{2} Q, \cdots, \gamma_{n_{1}} Q\right), m s k=\left(\gamma_{1}, \gamma_{2}, \cdots, \gamma_{n_{1}}\right)\right) .
\end{aligned}
$$

3. $\operatorname{Encrypt}\left(p k_{i_{1}},\left(i_{1}, i_{2}\right), m\right)$ : For a message $m \in \mathbb{G}_{T}$ and an index $\left(i_{1}, i_{2}\right) \in$ $\left\{1,2, \cdots, n_{1}\right\} \times\left\{1,2, \cdots, n_{2}\right\}$, randomly choose $r \in \mathbb{Z}_{q}$ and let $t^{\prime}=t+r \in \mathbb{Z}_{q}$. Then compute the ciphertext as $\mathcal{C}=\left(r Q, t^{\prime}\left(p k^{2}{ }_{i_{1}}+Q_{i_{2}}\right), m \cdot \hat{e}^{\prime \prime}\left(P_{n_{2}}, t^{\prime} Q_{1}\right)\right)=\left(c_{1}, c_{2}, c_{3}\right)$.

4. $\operatorname{Extract}(m s k=\gamma, \mathcal{S})$ : For the set $\mathcal{S}$ of indices $\left(j_{1}, j_{2}\right)$ the aggregate key is computed as $K_{\mathcal{S}}=\left(k_{\mathcal{S}}^{1}, k_{\mathcal{S}}^{2}, \cdots, k_{\mathcal{S}}^{n_{1}}\right)=$ $\left(\sum_{\left(1, j_{2}\right) \in \mathcal{S}} \gamma_{1} P_{n_{2}+1-j_{2}}, \sum_{\left(2, j_{2}\right) \in \mathcal{S}} \gamma_{2} P_{n_{2}+1-j_{2}}, \cdots, \sum_{\left(n_{1}, j_{2}\right) \in \mathcal{S}} \gamma_{n_{1}} P_{n_{2}+1-j_{2}}\right)$ and the dynamic access control parameter $U$ is computed as $t Q$. Thus the net aggregate key is $\left(K_{\mathcal{S}}, U\right)$ which is transmitted via a secure channel to users that have access rights to $\mathbb{S}$. Note that $k_{\mathcal{S}}^{j_{1}}=\sum_{\left(j_{1}, j_{2}\right) \in \mathcal{S}} \alpha^{n+1-j} p k^{1}{ }_{j_{1}}$ for $j_{1}=1,2, \cdots, n_{1}$.

5. $\operatorname{Decrypt}\left(K_{\mathcal{S}}, U, \mathcal{S},\left(i_{1}, i_{2}\right), \mathcal{C}=\left\{c_{1}, c_{2}, c_{3}\right\}\right)$ : If $\left(i_{1}, i_{2}\right) \notin \mathcal{S}$, output $\perp$. Otherwise return the message

$$
\hat{m}=c_{3} \frac{e^{\hat{\prime \prime}}\left(k_{\mathcal{S}}^{i_{1}}+\sum_{\left(i_{1}, j_{2}\right) \in \mathcal{S}, j_{2} \neq i_{2}} P_{\left.n_{2}+1-j_{2}+i_{2}, U+c_{1}\right)}\right.}{e^{\hat{\prime \prime}\left(\sum_{\left(i_{1}, j_{2}\right) \in \mathcal{S}} P_{n_{2}+1-j_{2}}, c_{2}\right)} .}
$$


The proof of correctness of this scheme is presented below.

$$
\begin{aligned}
& \hat{m}=c_{3} \frac{e^{\hat{\prime \prime}}\left(k_{\mathcal{S}}^{i_{1}}+\sum_{\left(i_{1}, j_{2}\right) \in \mathcal{S}, j_{2} \neq i_{2}} P_{n_{2}+1-j_{2}+i_{2}}, U+c_{1}\right)}{e^{\prime \prime}\left(\sum_{\left(i_{1}, j_{2}\right) \in \mathcal{S}} P_{n_{2}+1-j_{2}}, c_{2}\right)} \\
& =c_{3} \frac{e^{\prime \prime}\left(\sum_{\left(i_{1}, j_{2}\right) \in \mathcal{S}} \gamma_{i_{1}} P_{n_{2}+1-j_{2}}, t^{\prime} Q\right) e^{\prime \prime}\left(\sum_{\left(i_{1}, j_{2}\right) \in \mathcal{S}}\left(P_{n_{2}+1-j_{2}+i_{2}}\right)-P_{n_{2}+1}, t^{\prime} Q\right)}{e^{\hat{\prime \prime}}\left(\sum_{\left(i_{1}, j_{2}\right) \in \mathcal{S}} P_{n_{2}+1-j_{2}}, \gamma_{i_{1}}\left(t^{\prime} Q\right)\right) e^{\hat{\prime \prime}}\left(\sum_{\left(i_{1}, j_{2}\right) \in \mathcal{S}} P_{n_{2}+1-j_{2}}, \alpha^{i_{2}}\left(t^{\prime} Q\right)\right)} \\
& =c_{3} \frac{e^{\prime \prime}\left(\sum_{\left(i_{1}, j_{2}\right) \in \mathcal{S}} P_{n_{2}+1-j_{2}+i_{2}}, t^{\prime} Q\right)}{e^{\prime \prime}\left(P_{n_{2}+1}, t^{\prime} Q\right) e^{\prime \prime}\left(\sum_{\left(i_{1}, j_{2}\right) \in \mathcal{S}} P_{n_{2}+1-j_{2}+i_{2}}, t^{\prime} Q\right)} \\
& =m
\end{aligned}
$$

\subsection{Semantic Security of the Extended KAC}

The proof of security uses a reduced version of the extended KAC scheme, analogous to the reduced scheme used for proving the security of the generalized KAC. The adversarial model is also the assumed to be the same as for the generalized KAC. The proof uses the $(l, l)$-BDHE assumption proposed in 3.3. Let $\mathbb{G}_{1}$ and $\mathbb{G}_{2}$ be additive elliptic curve subgroups of prime order $q$, and $G_{T}$ be a multiplicative group of order $q$. Let $e^{\prime \prime}: \mathbb{G}_{1} \times \mathbb{G}_{2} \longrightarrow \mathbb{G}_{T}$ be a bilinear nondegenerate pairing. We claim that for any pair of positive integers $n_{2}, n^{\prime}\left(n^{\prime}>n_{2}\right)$ our proposed extension to the $n_{2}$-generalized reduced key-aggregate encryption scheme over elliptic curve subgroups is $\left(\tau, \epsilon, n^{\prime}\right)$ semantically secure if the decision $\left(\tau, \epsilon, n_{2}, n_{2}\right)$-BDHE assumption holds in $\left(\mathbb{G}_{1}, \mathbb{G}_{2}\right)$. We prove the claim below.

Proof: Let for a given input $n^{\prime}, \mathcal{A}$ be a $\tau$-time adversary that has advantage greater than $\epsilon$ for the reduced scheme parameterized with a given $n_{2}$. We build an algorithm $\mathcal{B}$ that has advantage at least $\epsilon$ in solving the $\left(n_{2}, n_{2}\right)$-BDHE problem in $\mathbb{G}$. Algorithm $\mathcal{B}$ takes as input a random $\left(n_{2}, n_{2}\right)$-BDHE challenge $\left(P, Q, H, Y_{\left(P, \alpha, n_{2}\right), Y_{Q, \alpha, n_{2}}^{\prime}}, Z\right)$ where $Z$ is either $\hat{e}^{\prime \prime}\left(P_{n_{2}+1}, H\right)$ or a random value in $\mathbb{G}_{T}$. Algorithm $\mathcal{B}$ proceeds as follows.

1. Init: Algorithm $\mathcal{B}$ runs $\mathcal{A}$ and receives the set $\mathcal{S}$ of ciphertext classes that $\mathcal{A}$ wishes to be challenged on. For each ciphertext class $\left(i_{1}, i_{2}\right) \in \mathcal{S}, \mathcal{B}$ performs the SetUp- $\left(\mathbf{i}_{1}, \mathbf{i}_{2}\right)$, Challenge- $\left(\mathbf{i}_{1}, \mathbf{i}_{2}\right)$ and Guess- $\left(\mathbf{i}_{1}, \mathbf{i}_{2}\right)$ steps. Note that the number of iterations is polynomial in $|S|$.

2. SetUp- $\left(\mathbf{i}_{1}, \mathbf{i}_{2}\right): \mathcal{B}$ should generate the public param, public keys $P K^{1}, P K^{2}$, the access parameter $U$, and the aggregate key $K_{\overline{\mathcal{S}}}$. For the iteration corresponding to ciphertext class $\left(i_{1}, i_{2}\right)$, they are generated as follows.

- param is set as $\left(P, Q, Y_{P, \alpha, n_{2}}, Y_{Q, \alpha, n_{2}}^{\prime}\right)$.

- Randomly generate $u_{1}, u_{2}, \cdots, u_{n_{1}} \in \mathbb{Z}_{q}$. Then, set $P K^{1}=\left(p k^{1}{ }_{1}, p k_{2}^{1}, \cdots, p k_{n_{1}}^{1}\right)$, where $p k^{1}{ }_{j_{1}}$ is set as $u_{j_{1}} P-P_{i_{2}}$ for $j_{1}=$ $1,2, \cdots, n_{1}$, and set $P K^{2}=\left(p k^{2}{ }_{1}, p k_{2}^{2}, \cdots, p k^{2}{ }_{n_{1}}\right)$, where $p k_{j_{1}}^{2}$ is set as $u_{j_{1}} Q-Q_{i_{2}}$ for $j_{1}=$ $1,2, \cdots, n_{1}$

- $K_{\overline{\mathcal{S}}}$ is set as $\left(k_{\overline{\mathcal{S}}}^{1}, k_{\overline{\mathcal{S}}}^{2}, \cdots, k_{\overline{\mathcal{S}}}^{n_{1}}\right)$ where $k_{\overline{\mathcal{S}}}^{j_{1}}$ $=\sum_{\left(j_{1}, j_{2}\right) \notin \mathcal{S}}\left(u P_{n_{2}+1-j_{2}}-\left(P_{n_{2}+1-j_{2}+i_{2}}\right)\right)$ for $j_{1}=1,2, \cdots, n_{1}$. Note that 
this implies $k_{\mathcal{\mathcal { S }}}^{j_{1}}=\sum_{\left(j_{1}, j_{2}\right) \notin \mathcal{S}} \alpha^{n_{2}+1-j_{2}} p k^{1}{ }_{j_{1}}$, as is supposed to be as per the scheme specification. Note that $\mathcal{B}$ knows that $\left(i_{1}, i_{2}\right) \notin \overline{\mathcal{S}}$, and hence has all the resources to compute this aggregate key for $\overline{\mathcal{S}}$.

- $U$ is set as some random element in $\mathbb{G}_{2}$.

Note that since $P, Q, \alpha, U$ and the $u_{j_{1}}$ values are chosen uniformly at random, the public key has an identical distribution to that in the actual construction.

3. Challenge- $\left(\mathbf{i}_{1}, \mathbf{i}_{2}\right)$ : To generate the challenge for the ciphertext class $\left(i_{1}, i_{2}\right)$, $\mathcal{B}$ computes $\left(c_{1}, c_{2}\right)$ as $\left(H-U, u_{i_{1}} H\right)$. It then randomly chooses a bit $b \in(0,1)$ and sets $K_{b}$ as $Z$ and $K_{1-b}$ as a random element in $\mathbb{G}_{T}$. The challenge given to $\mathcal{A}$ is $\left(\left(c_{1}, c_{2}\right), K_{0}, K_{1}\right)$.

We claim that when $Z=\hat{e}^{\prime \prime}\left(P_{n_{2}+1}, H\right)$ (i.e. the input to $\mathcal{B}$ is a $n_{2}$-BDHE tuple), then $\left(\left(c_{1}, c_{2}\right), K_{0}, K_{1}\right)$ is a valid challenge to $A$. We prove this claim here. we point out that $Q$ is a generator of $\mathbb{G}_{2}$ and so $H=t^{\prime} P$ for some $t^{\prime} \in \mathbb{Z}_{q}$. Putting $H$ as $t^{\prime} Q$ gives us the following:

$-U=t Q$ for some $t \in \mathbb{Z}_{q}$

- $c_{1}=H-U=\left(t^{\prime}-t\right) Q=r Q$ where $r=t^{\prime}-t$

$-c_{2}=u_{i_{1}} H=\left(u_{i_{1}}\right) t^{\prime} Q=t^{\prime}\left(u_{i_{1}} Q\right)=t^{\prime}\left(u_{i_{1}} Q-Q_{i_{2}}+Q_{i_{2}}\right)=t^{\prime}\left(p k_{i_{1}}^{2}+Q_{i_{2}}\right)$

- $K_{b}=Z=\hat{e}^{\prime}\left(P_{n_{2}+1}, H\right)=\hat{e}^{\prime}\left(P_{n_{2}+1}, t^{\prime} Q\right)$

On the other hand, if $Z$ is a random element in $\mathbb{G}_{T}$ (i.e. the input to $\mathcal{B}$ is a random tuple), then $K_{0}$ and $K_{1}$ are just random independent elements of $\mathbb{G}_{T}$.

4. Guess-(i $\left.\mathbf{i}_{1}, \mathbf{i}_{2}\right)$ : The adversary $\mathcal{A}$ outputs a guess $b^{\prime}$ of $b$. If $b^{\prime}=b, \mathcal{B}$ outputs 0 (indicating that $Z=\hat{e}^{\prime \prime}\left(P_{n+1}, H\right)$ ), and terminates. Otherwise, it goes for the next ciphertext class in $\mathcal{S}$.

If after $|\mathcal{S}|$ iterations, $b^{\prime} \neq b$ for each ciphertext class $\left(i_{1}, i_{2}\right) \in \mathcal{S}$, the algorithm $\mathcal{B}$ outputs 1 (indicating that $Z$ is random in $\mathbb{G}_{T}$ ). We now analyze the probability that $\mathcal{B}$ gives a correct output. If $\left(P, H, Y_{\left(P, \alpha, n_{2}\right)}, Z\right)$ is sampled from $R^{\prime}$-BDHE, $\operatorname{Pr}\left[\mathcal{B}\left(G, H, Y_{\left(P, \alpha, n_{2}\right)}, Z\right)=0\right]=\frac{1}{2}$, while if $\left(P, H, Y_{\left(P, \alpha, n_{2}\right)}, Z\right)$ is sampled from $L^{\prime}$-BDHE, $\left|\operatorname{Pr}\left[\mathcal{B}\left(G, H, Y_{\left(P, \alpha, n_{2}\right)}, Z\right)\right]-\frac{1}{2}\right| \geq \epsilon$. So, the probability that $\mathcal{B}$ outputs correctly is at least $1-\left(\frac{1}{2}-\epsilon\right)^{|\mathcal{S}|} \geq \frac{1}{2}+\epsilon$. Thus $\mathcal{B}$ has advantage at least $\epsilon$ in solving the $\left(n_{2}, n_{2}\right)$-BDHE problem. This concludes the proof.

\section{Experimental Results Using Tate pairings}

In this section we present experimental results from our implementations of the extended generalized scheme using Tate pairings on BN-curves using 256 bit primes [33]. All our experiments have been carried out on an AMD Opteron (TM) Processor $6272 \times 16$ with a clock frequency $1.4 \mathrm{GHz}$. The details of our implementations of Tate Pairings are summarized in Appendix A.

\subsection{Space and Time Complexities}

Table 2 summarizes the space requirements for various parameters of the scheme for different values of $\left(n_{1}, n_{2}\right)$. The results have been averaged over 100 randomly 
Table 2: Space Complexities

\begin{tabular}{|c|c|c|c|c|c|c|c|}
\hline$n_{1}$ & $n_{2}$ & $\begin{array}{c}\text { param } \\
\text { (in bytes) }\end{array}$ & $\begin{array}{c}P K \\
\text { (in bytes) }\end{array}$ & $\begin{array}{c}m s k \\
\text { (in bytes) }\end{array}$ & $\begin{array}{c}K_{\mathcal{S}} \\
\text { (in bytes) }\end{array}$ & $\begin{array}{c}U \\
\text { (in bytes) }\end{array}$ & $\begin{array}{c}\text { Total } \\
\text { (in KB) }\end{array}$ \\
\hline \hline 1 & 100 & 16112 & 144 & 40 & 72 & 64 & 16.046875 \\
\hline 2 & 50 & 8112 & 240 & 56 & 120 & 64 & 8.390625 \\
\hline 4 & 25 & 4112 & 432 & 88 & 216 & 64 & 4.796875 \\
\hline 5 & 20 & 3312 & 528 & 104 & 264 & 64 & 4.171875 \\
\hline $\mathbf{1 0}$ & $\mathbf{1 0}$ & $\mathbf{1 7 1 2}$ & $\mathbf{1 0 0 8}$ & $\mathbf{1 8 4}$ & $\mathbf{5 0 4}$ & $\mathbf{6 4}$ & $\mathbf{3 . 3 9 0 6 2 5}$ \\
\hline 20 & 5 & 912 & 1968 & 344 & 984 & 64 & 4.171875 \\
\hline 25 & 4 & 752 & 2448 & 424 & 1224 & 64 & 4.796875 \\
\hline 50 & 2 & 432 & 4848 & 824 & 2424 & 64 & 8.390625 \\
\hline 100 & 1 & 272 & 9648 & 1624 & 4824 & 64 & 16.046875 \\
\hline \hline
\end{tabular}

Table 3: Time Complexities

\begin{tabular}{|c|c|c|c|c|c|c|c|}
\hline$n_{1}$ & $n_{2}$ & $\begin{array}{c}\text { SetUp } \\
\text { (in clock cycles) }\end{array}$ & $\begin{array}{c}\text { KeyGen } \\
\text { (in clock cycles) }\end{array}$ & $\begin{array}{c}\text { Encrypt } \\
\text { (in clock cycles) }\end{array}$ & $\begin{array}{c}\text { Extract } \\
\text { (in clock cycles) }\end{array}$ & $\begin{array}{c}\text { Decrypt } \\
\text { (in clock cycles) }\end{array}$ & $\begin{array}{c}\text { Total } \\
\text { (in clock cycles) }\end{array}$ \\
\hline \hline 1 & 100 & 2920000 & 10000 & 7932000 & 47000 & 16095000 & 27004100 \\
\hline 2 & 50 & 1410000 & 30000 & 8065000 & 53000 & 16110000 & 25668000 \\
\hline 4 & 25 & 690000 & 60000 & 8130000 & 81000 & 16284000 & 25245000 \\
\hline 5 & 20 & 590000 & 70000 & 8091000 & 96000 & 16379000 & 25226000 \\
\hline $\mathbf{1 0}$ & $\mathbf{1 0}$ & $\mathbf{2 8 0 0 0 0}$ & $\mathbf{1 4 0 0 0 0}$ & $\mathbf{7 9 5 7 0 0 0}$ & $\mathbf{1 7 0 0 0 0}$ & $\mathbf{1 6 0 4 9 0 0 0}$ & $\mathbf{2 5 1 3 6 0 0 0}$ \\
\hline 20 & 5 & 130000 & 270000 & 8070000 & 320000 & 16361000 & 25151000 \\
\hline 25 & 4 & 120000 & 350000 & 8256000 & 370000 & 16239000 & 25836000 \\
\hline 50 & 2 & 50000 & 680000 & 8265000 & 712000 & 16398000 & 26105000 \\
\hline 100 & 1 & 30000 & 1360000 & 8201000 & 1315000 & 16142000 & 27048000 \\
\hline
\end{tabular}

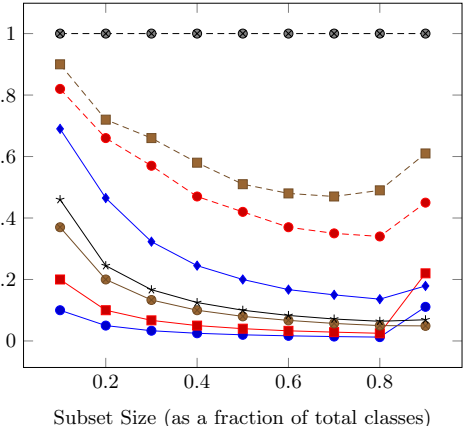

$$
\begin{aligned}
& \bullet \quad n_{1}=1, n_{2}=100 \\
& n_{1}=2, n_{2}=50 \\
& n_{1}=4, n_{2}=25 \\
& n_{1}=5, n_{2}=20 \\
& n_{1}=10, n_{2}=10 \\
& n_{1}=20, n_{2}=5 \\
& \text { - } \quad n_{1}=25, n_{2}=4 \\
& -\otimes\left(n_{1}=50, n_{2}=2\right) \&\left(n_{1}=100, n_{2}=1\right)
\end{aligned}
$$

Fig. 4: Key Size ratio - Proposed Aggregate Scheme vs Hierarchical Scheme

chosen subsets of the $n=100$ ciphertext classes. Table 3 summarizes the time complexity for various operations of the scheme for different values of $\left(n_{1}, n_{2}\right)$. The results have been averaged over 100 randomly chosen subsets of the $n=100$ ciphertext classes. The encryption and decryption operation complexities are further averaged over 10 message transmissions corresponding to each subset. We point out that both the overall space and time requirements are minimum for $n_{1}=n_{2}=10=\sqrt{ } n$, which proves the usefulnesss of the generaalization.

\subsection{Comparison with Hierarchy Based Schemes}

Next, we compare specifically the key size required for the proposed extended scheme, for different values of $n_{1}$ and $n_{2}$ (again corresponding to $n=100$ ), with that required for a hierarchical encryption construction [9]. Since our scheme 


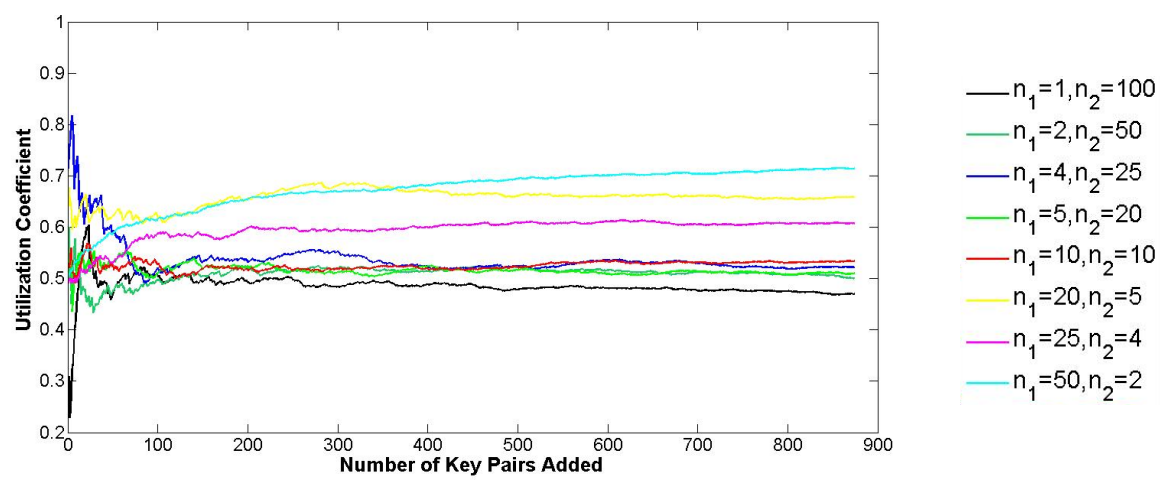

Fig. 6: Utilization coefficient vs Newly Registered Keys

uses a hierarchy depth of 2 , we use the same for the hierarchical construction as well, with $n_{1}$ nodes in level 0 , and $n_{2}$ level 1 nodes in the subtree rooted at each level 0 node. Figure 4 summarizes the findings. Evidently, lower the value of $n_{1}$, better the key aggregation, hence lower the ratio.

\subsection{Utilization Coefficient Comparison}

Finally we compare the utilization-coefficient of the extended scheme for various values of $n_{1}$ and $n_{2}$ (corresponding to $n=100$ ) with increase in the number of registered key pairs $l$, where each key pair increases the number of classes by $n_{2}$. We leave out the configuration $n_{1}=n, n_{2}=1$ because that always leads to an utilization coefficient of 1 but is impractical due to huge space requirements. Figure 6 demonstrates that that beyond a certain value of $l$, the combination $(1, n)$ proposed in [3] has a lower utilization coefficient that all other combinations of $\left(n_{1}, n_{2}\right)$ for a given $n$. This emphasizes the advantage of making the choice of $\left(n_{1}, n_{2}\right)$ flexible.

\section{Conclusions and Future Work}

In this paper, we have proposed a secure and dynamic key aggregate encryption scheme for online data sharing. Our scheme allows data owners to delegate users with access rights to multiple ciphertext classes using a single decryption key that combines the decrypting power of individual keys corresponding to each ciphertext class. Unlike existing key aggregate schemes that are static in their access right delegation policies, our scheme allows data owners to dynamically revoke one or more users' access rights without having to change either the public or the private parameters/keys. The use of bilinear pairings over additive elliptic curve subgroups in our scheme helps achieve massive reductions in key and ciphertext sizes over existing schemes that use multiplicative groups. We pointed out that a possible criticism of this scheme is that the number of 
classes is pre-defined to some fixed $n$. To deal with this issue, we next proposed a generalized two-level construction of the basic scheme that runs $n_{1}$ instances of the basic scheme in parallel, with each instance handling key aggregation for $n_{2}$ ciphertext classes. This scheme provides two major advantages. First of all, it allows dynamic extension of ciphertext classes by registering of new public key-private key pairs without affecting other system parameters. Secondly, it provides a wide range of choices for $n_{1}$ and $n_{2}$ that allows efficient utilization of ciphertext classes while also achieving optimum space and time complexities. Finally, we extend the generalized scheme to allow the use of popular and efficiently implementable bilinear pairings in literature such as Tate Pairings that operate on multiple elliptic curve subgroups instead of one. Each of the three proposed schemes have been proven to be semantically secure. Experimental studies have demonstrated the superiority of our proposed scheme over existing ones in terms of key size as well as efficient utilization of ciphertext classes. A possible future work is to make the proposed schemes secure against chosen ciphertext attacks.

\section{References}

1. Cong Wang, Sherman S.-M. Chow, Qian Wang, Kui Ren, and Wenjing Lou. Privacy-preserving public auditing for secure cloud storage. Cryptology ePrint Archive, Report 2009/579, 2009. http://eprint.iacr.org/.

2. Sherman SM Chow, Cheng-Kang Chu, Xinyi Huang, Jianying Zhou, and Robert H Deng. Dynamic secure cloud storage with provenance. In Cryptography and Security: From Theory to Applications, pages 442-464. Springer, 2012.

3. Cheng-Kang Chu, Sherman SM Chow, Wen-Guey Tzeng, Jianying Zhou, and Robert H Deng. Key-aggregate cryptosystem for scalable data sharing in cloud storage. Parallel and Distributed Systems, IEEE Transactions on, 25(2):468-477, 2014.

4. Dan Boneh, Craig Gentry, and Brent Waters. Collusion resistant broadcast encryption with short ciphertexts and private keys. In Advances in Cryptology-CRYPTO 2005, pages 258-275. Springer, 2005.

5. Selim G Akl and Peter D Taylor. Cryptographic solution to a problem of access control in a hierarchy. ACM Transactions on Computer Systems (TOCS), 1(3):239248, 1983.

6. Gerald C Chick and Stafford E Tavares. Flexible access control with master keys. In Advances in CryptologyCRYPTO89 Proceedings, pages 316-322. Springer, 1990.

7. Wen-Guey Tzeng. A time-bound cryptographic key assignment scheme for access control in a hierarchy. Knowledge and Data Engineering, IEEE Transactions on, 14(1):182-188, 2002.

8. Giuseppe Ateniese, Alfredo De Santis, Anna Lisa Ferrara, and Barbara Masucci. Provably-secure time-bound hierarchical key assignment schemes. Journal of cryptology, 25(2):243-270, 2012.

9. Ravinderpal S Sandhu. Cryptographic implementation of a tree hierarchy for access control. Information Processing Letters, 27(2):95-98, 1988.

10. Yan Sun and KJ Liu. Scalable hierarchical access control in secure group communications. In INFOCOM 2004. Twenty-third AnnualJoint Conference of the IEEE Computer and Communications Societies, volume 2, pages 1296-1306. IEEE, 2004. 
11. William C King and Bjorn Hjelm. Centralized key management, March 242015. US Patent 8,990,555.

12. Mikhail J Atallah, Marina Blanton, Nelly Fazio, and Keith B Frikken. Dynamic and efficient key management for access hierarchies. ACM Transactions on Information and System Security (TISSEC), 12(3):18, 2009.

13. Josh Benaloh, Melissa Chase, Eric Horvitz, and Kristin Lauter. Patient controlled encryption: ensuring privacy of electronic medical records. In Proceedings of the 2009 ACM workshop on Cloud computing security, pages 103-114. ACM, 2009.

14. Josh Benaloh. Key compression and its application to digital fingerprinting. Technical report, Technical Report Technical Report, Microsoft Research, 2009.

15. Basel Alomair and Radha Poovendran. Information theoretically secure encryption with almost free authentication. J. UCS, 15(15):2937-2956, 2009.

16. Adi Shamir. Identity-based cryptosystems and signature schemes. In Advances in cryptology, pages 47-53. Springer, 1985.

17. Dan Boneh and Matthew Franklin. Identity-based encryption from the weil pairing. SIAM Journal on Computing, 32(3):586-615, 2003.

18. Clifford Cocks. An identity based encryption scheme based on quadratic residues. In Cryptography and coding, pages 360-363. Springer, 2001.

19. Fuchun Guo, Yi Mu, and Zhide Chen. Identity-based encryption: how to decrypt multiple ciphertexts using a single decryption key. In Pairing-Based CryptographyPairing 2007, pages 392-406. Springer, 2007.

20. Fuchun Guo, Yi Mu, Zhide Chen, and Li Xu. Multi-identity single-key decryption without random oracles. In Information Security and Cryptology, pages 384-398. Springer, 2008.

21. Amit Sahai and Brent Waters. Fuzzy identity-based encryption. In Advances in Cryptology-EUROCRYPT 2005, pages 457-473. Springer, 2005.

22. Vipul Goyal, Omkant Pandey, Amit Sahai, and Brent Waters. Attribute-based encryption for fine-grained access control of encrypted data. In Proceedings of the 13th ACM conference on Computer and communications security, pages 89-98. Acm, 2006.

23. John Bethencourt, Amit Sahai, and Brent Waters. Ciphertext-policy attributebased encryption. In Security and Privacy, 200\%. SP'0\%. IEEE Symposium on, pages 321-334. IEEE, 2007.

24. Melissa Chase. Multi-authority attribute based encryption. In Theory of cryptography, pages 515-534. Springer, 2007.

25. Ming Li, Shucheng Yu, Yao Zheng, Kui Ren, and Wenjing Lou. Scalable and secure sharing of personal health records in cloud computing using attribute-based encryption. Parallel and Distributed Systems, IEEE Transactions on, 24(1):131$143,2013$.

26. Giuseppe Ateniese, Kevin Fu, Matthew Green, and Susan Hohenberger. Improved proxy re-encryption schemes with applications to secure distributed storage. ACM Transactions on Information and System Security (TISSEC), 9(1):1-30, 2006.

27. Qin Liu, Chiu C Tan, Jie Wu, and Guojun Wang. Reliable re-encryption in unreliable clouds. In Global Telecommunications Conference (GLOBECOM 2011), 2011 IEEE, pages 1-5. IEEE, 2011.

28. Victor Miller. Use of elliptic curves in cryptography. In Advances in CryptologyCRYPTO85 Proceedings, pages 417-426. Springer, 1986.

29. Gerhard Frey and Hans-Georg Rück. A remark concerning -divisibility and the discrete logarithm in the divisor class group of curves. Mathematics of computation, 62(206):865-874, 1994. 
30. Florian Hess, Nigel P Smart, and Frederik Vercauteren. The eta pairing revisited. Information Theory, IEEE Transactions on, 52(10):4595-4602, 2006.

31. Chang-An Zhao, Fangguo Zhang, and Jiwu Huang. A note on the ate pairing. International Journal of Information Security, 7(6):379-382, 2008.

32. Leonardo B Oliveira, Diego F Aranha, Eduardo Morais, Felipe Daguano, Julio López, and Ricardo Dahab. Tinytate: Computing the tate pairing in resourceconstrained sensor nodes. In Network Computing and Applications, 2007. NCA 200\%. Sixth IEEE International Symposium on, pages 318-323. IEEE, 2007.

33. Santosh Ghosh, Debdeep Mukhopadhyay, and Dipanwita Roychowdhury. Secure dual-core cryptoprocessor for pairings over barreto-naehrig curves on fpga platform. Very Large Scale Integration (VLSI) Systems, IEEE Transactions on, 21(3):434-442, 2013.

34. Paulo SLM Barreto and Michael Naehrig. Pairing-friendly elliptic curves of prime order. In Selected areas in cryptography, pages 319-331. Springer, 2006.

35. Paulo S. L. M. Barreto and Michael Naehrig. Pairing-friendly elliptic curves of prime order. Cryptology ePrint Archive, Report 2005/133, 2005. http://eprint.iacr.org/.

36. Augusto Jun Devegili, Michael Scott, and Ricardo Dahab. Implementing cryptographic pairings over barreto-naehrig curves. In Pairing-Based CryptographyPairing 2007, pages 197-207. Springer, 2007. 


\section{A Implementation of Tate pairings Using BN Curves}

\section{A.1 The Tate pairing}

We first provide a brief overview of the Tate pairing. Let $\mathbb{K}$ be a field of prime order $p$, and let an elliptic curve $E(K)$ over $\mathbb{K}$ be defined by the Weierstrass [28] equation. Also, Let $\bar{K}=F_{p^{k}}$ be the smallest extension field of $K=F_{p}$ that contains the $q^{t h}$ roots of unity. We refer to $k$ as the embedding degree with respect to $K$ and $q$. Further, we refer to the set of $q$-torsion points on the elliptic curve as $E(\bar{K})[q]$ ( $q$-torsion points essentially have order $q$ ). Before defining the Tate pairing, we briefly state the Miller's function [28]. Let $[a] P$ denote the multiplication of a point $P \in E$ by a scalar $a \in \mathbb{Z}$ (equivalent to adding $P a$ times), and let $\mathcal{O} \in E$ denote the point at infinity. A Miller function is any rational function on $E$ that has a divisor of the form

$$
\left(f_{q, P}\right)=q(P)-([q] P)-(q-1) \mathcal{O} .
$$

A Miller function has $q$ zeros at $P$, one pole at $[q] P$ and $q-1$ poles at $\mathcal{O}$. For every point $Q \neq P,[q] P, I$, we have $\left(f_{q, P}\right) \in \bar{K}^{*}$. We now define the Tate pairing over elliptic curves.

The Tate pairing $e_{T}: \mathbb{G}_{1} \times \mathbb{G}_{2} \longrightarrow \mathbb{G}_{T}$ is a well-defined, non-degenerate, bilinear pairing with $\mathbb{G}_{1}=E(K)[q], \mathbb{G}_{2}=E(\bar{K}) / q E(\bar{K})$, and $\mathbb{G}_{T}=\bar{K}^{*} /\left(\bar{K}^{*}\right)^{q}$. Let $P \in E(\bar{K})[q]$ and $Q \in E(\bar{K}) / q E(\bar{K})$. Then the Tate pairing of $P, Q$ is computed as

$$
e_{T}(P, Q)=f_{q, P}(Q)^{\frac{p^{k}-1}{q}}
$$

Properties: Tate pairing satisfies following properties that make the pairing suitable for use in cryptography.

- Well defined: $e_{T}(\mathcal{O}, Q)=1$ for all $Q \in E(\bar{K})$ and $e_{T}(P, Q) \in\left(\bar{K}^{*}\right)^{q}$ for all $P \in E(\bar{K})[q]$ and all $Q \in q E(\bar{K})$.

- Bilinearity: For all $P, P_{1}, P_{2} \in E(\bar{K})[q]$ and $Q, Q_{1}, Q_{2} \in E(\bar{K})$, we have

$-e_{T}\left(P_{1}+P_{2}, Q\right)=e_{T}\left(P_{1}, Q\right) \cdot e_{T}\left(P_{2}, Q\right)$.

$-e_{T}\left(P, Q_{1}+Q_{2}\right)=e_{T}\left(P, Q_{1}\right) \cdot e_{T}\left(P, Q_{2}\right)$.

- Non-degeneracy: For each point $E(\bar{K})[q] \backslash \mathcal{O}$ there is some point $Q \in E(\bar{K})$ such that $e_{T}(P, Q) \notin(\bar{K})^{q}$.

\section{A.2 Pairing Friendly Curves}

Barreto and Naehrig [34] developed a method for constructing a method for constructing pairing-friendly elliptic curves over prime fields, with prime order and embedding degree $k=12$. The equation of the curve is $E: y^{2}=x^{3}+b$, with $b \neq 0$. The trace (of Frobenius) of the curve, the curve order and the 
characteristic of $\mathbb{F}_{p}$ are parameterized as:

$$
\begin{aligned}
t(x) & =6 x^{2}+1 \\
n(x) & =36 x^{4}-36 x^{3}+18 x^{2}-6 x+1 \\
p(x) & =36 x^{4}-36 x^{3}+24 x^{2}-6 x+1
\end{aligned}
$$

respectively. Such a curve is often referred to in literature a Barreto-Naehrig or BN curve. Since every point on the BN curve has order $n$, the value of $q$ (a large prime dividing the curve order) can be taken to be the same as $n$.

Suitability of Barreto-Naehrig curves: BN curves are especially well suited for the 128-bit security level. This is because, if $p$ is 256-bit prime, then the Pollards rho method for computing discrete logarithms in $E\left(\mathbb{F}_{p}\right)$ has running time approximately $2^{128}$, as does the number field sieve algorithm for computing discrete logarithms in the extension field $\mathbb{F}_{p^{12}}$. The biggest advantage of using $\mathrm{BN}$ curves is that they admit sextic twists with degree six, implying that there exists a distortion map between $\mathbb{F}_{p^{2}}$ and $\mathbb{F}_{p^{1} 2}$. This is of great advantage from the computational point of view since many computations can now be restricted to the field $\mathbb{F}_{p^{2}}$. The other advantage of using BN curves is their flexibility in terms of order of the prime $p$. Barreto and Naehrig have defined in [34] a whole family of $\mathrm{BN}$ curves to choose from, corresponding to primes of any given order.

Barreto-Naehrig curve used in implementation The BN curve used in our implementation for 256 bit primes is given by

$$
E: Y^{2}=X^{3}+3
$$

with BN parameter $x=6000000000001 F 2 D$ (in hexadecimal). The corresponding prime $p(x)=36 x^{4}-36 x^{3}+24 x^{2}-6 x+1$ is a 256-bit prime of Hamming weight $87, n(x)=36 x^{4}-36 x^{3}+18 x^{2}-6 x+1$ is 256-bit prime of Hamming weight 91 , and $t-1=p-r=6 z^{2}+1$ is a 128-bit integer of Hamming weight 28(here $t=p+1-r$ is the trace of $E$ ). Note that the choice of $p$ is made such that $p \equiv 7(\bmod 8), p \equiv 4(\bmod 9) p \equiv 1(\bmod 6)$. The reason for this is as follows.

1. The first condition ensures that -2 is a quadratic non-residue.

2. The second condition ensures efficient computation of cube roots [35].

3. The third condition ensures that there exists $\xi \in \mathbb{F}_{p^{2}}$ such that $W^{6}-\xi$ is irreducible over $\mathbb{F}_{p^{2}}[W]$.

\section{A.3 The Finite Field Extensions}

As per the proposition in [36], we construct the extension field $\mathbb{F}_{p^{12}}$ using the following tower field extensions: 
1. $\mathbb{F}_{p^{2}}=\mathbb{F}_{p}[u] /\left(u^{2}+2\right)$,

2. $\mathbb{F}_{p^{6}}=\mathbb{F}_{p^{2}}[v] /\left(v^{3}-\xi\right)$ where $\xi=-u-1$, and

3. $\mathbb{F}_{p^{12}}=\mathbb{F}_{p^{6}}[w] /\left(w^{2}-v\right)$.

The quadratic/cubic non-residues and reduction polynomials are detailed in Table 4 for $a_{0}, a_{1} \in \mathbb{F}_{p}, b_{0}, b_{1}, b_{2} \in \mathbb{F}_{p^{2}}$, and $c_{0}, c_{1} \in \mathbb{F}_{p^{6}}$.

Table 4: Extension fields

\begin{tabular}{|c|c|c|c|}
\hline Extension & Non-Residue & Construction & Representation \\
\hline $\mathbb{F}_{p^{2}}$ & $\beta=-2$ & $\mathbb{F}_{p}[X] /\left(X^{2}-\beta\right)$ & $a=a_{0}+a_{1} X$ \\
$\mathbb{F}_{p^{6}}$ & $\xi=-1 \sqrt{\beta}$ & $\mathbb{F}_{p^{2}}[Y] /\left(Y^{3}-\xi\right)$ & $b=b_{0}+b_{1} Y+b_{2} Y^{2}$ \\
$\mathbb{F}_{p^{12}}$ & $\xi^{\prime}=\sqrt[3]{\xi}$ & $\mathbb{F}_{p^{6}}[Z] /\left(Z^{2}-\xi^{\prime}\right)$ & $c=c_{0}+c_{1} Z$ \\
\hline
\end{tabular}

\section{A.4 The Actual Implementation}

The computation of the Tate pairing can be broadly divided into two major parts - the Miller's algorithm and the final exponentiation. A detailed implementation of the Miller's algorithm has been presented in [33] and we use the same for our experiments. The final exponentiation can also be efficiently implemented using the following factorization.

$$
\begin{aligned}
f^{\frac{p^{12}-1}{q}} & =f^{\left(p^{6}-1\right) \cdot \frac{p^{6}+1}{p^{4}-p^{2}+1} \cdot \frac{p^{4}-p^{2}+1}{q}} \\
& =\left(\left(f^{p^{6}-1}\right)^{p^{2}+1}\right)^{\frac{p^{4}-p^{2}+1}{q}}
\end{aligned}
$$

